ESAIM: PROCEEDINGS AND SURVEYS, December 2020, Vol. 69, p. 1-23

Philippe Helluy, Jean-Marc Hérard, Nicolas Seguin

\title{
LUBRICATION AND SHALLOW-WATER SYSTEMS BERNIS-FRIEDMAN AND BD ENTROPIES
}

\author{
Didier Bresch ${ }^{1}$, Mathieu Colin $^{2}$, Khawla Msheik ${ }^{3}$, Pascal Noble $^{4}$ and Xi Song ${ }^{5}$
}

\begin{abstract}
This paper concerns the results recently announced by the authors, in C.R. Acad. Sciences Maths volume 357, Issue 1, 1-6 (2019), which make the link between the BD entropy introduced by D. Bresch and B. Desjardins for the viscous shallow-water equations and the Bernis-Friedman (called $\mathrm{BF}$ in our paper) dissipative entropy introduced to study the lubrication equations. More precisely different dissipative BF entropies are obtained from the BD entropies playing with drag terms and capillarity formula for viscous shallow water type equations. This is the main idea in the paper which makes the link between two communities. The limit processes employ the standard compactness arguments taking care of the control in the drag terms. It allows in one dimension for instance to prove global existence of nonnegative weak solutions for lubrication equations starting from the global existence of nonnegative weak solutions for appropriate viscous shallow-water equations (for which we refer to appropriate references). It also allows to prove global existence of nonnegative weak solutions for fourth-order equation including the Derrida-Lebowitz-Speer-Spohn equation starting from compressible Navier-Stokes type equations.
\end{abstract}

Keywords. Lubrication and Shallow-water models, BD and BF entropies, Global weak solutions AMS subject classification: 35Q35, 35B25, 76N10,

\section{INTRODUCTION}

In a one-dimensional periodic torus domain $\Omega=\mathbb{T}$, lubrication type models with general representations of the surface tension and the second order dissipative term reads

$$
\partial_{t} h+\partial_{x}\left(\frac{1}{\alpha W_{e}} F(h) \partial_{x}^{3} h-\frac{1}{\alpha F r^{2}} D(h) \partial_{x} h\right)=0 .
$$

with the initial condition

$$
\left.h\right|_{t=0}=h_{0} \geq 0 \text { where } \int_{\Omega} h_{0}=M>0 .
$$

Note that the coefficients $M$ (total mass), $\alpha$ (drag term coefficient), We (Weissenberg number) and Fr (Froude number) are given positive coefficients and the nonnegative functions $s \mapsto F(s)$ and $s \mapsto D(s)$ depends on the applications. In their paper [1], Bernis and Friedman proved the existence of a weak solution for the higher

1 Laboratoire de Mathématiques UMR5127 CNRS, Université Savoie Mont-Blanc; e-mail: Didier.Bresch@univ-smb.fr

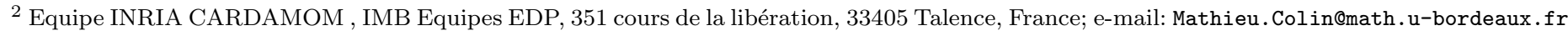

3 Laboratoire de Mathématiques UMR5127 CNRS, Université Savoie Mont-Blanc; e-mail: khawla.msheik@univ-smb.fr

${ }^{4}$ IMT, INSA Toulouse, 135 avenue de Rangueil, 31077 Toulouse Cedex 9, France; e-mail: pascal .noble@math.univ-toulouse.fr

5 e-mail: xi.song@hotmail.com

(C) EDP Sciences, SMAI 2020

This is an Open Access article distributed under the terms of the Creative Commons Attribution License (http://creativecommons.org/licenses/by/4.0), which permits unrestricted use, distribution, and reproduction in any medium, provided the original work is properly cited. 
order nonlinear degenerate parabolic equations with $F(h)=h^{n}$ and $D(h)=0$ with $n>1$ and suggested a new entropy inequality- referred to by BF entropy- which provides additional estimates serving for increasing the regularity of the weak solution obtained. This paper has been the starting point of plenty of important results for instance concerning the general class of $F(h)=h^{n}$ and $D(h)=h^{m}$ for some coeffficients $m$ and $n$, the interested reader is referred to [2], [1], 3] and [12] and references therein. It is important to explain what is the BF entropy and how to get it formally: A regularization process is used to justify it. Let us define the functionals

$$
g(s)=-\int_{s}^{A} \frac{1}{F(r)} d r, \quad G(s)=-\int_{s}^{A} g(r) d r,
$$

with A being an integer such that $A \geq \max |h(x, t)|$. According to Bernis and Friedman, we multiply (1) by $G^{\prime}(h)$

$$
\int_{\Omega} G^{\prime}(h) \partial_{t} h d x+\int_{\Omega} \partial_{x}\left(F(h) \partial_{x}^{3} h\right) G^{\prime}(h) d x-\int_{\Omega} \partial_{x}\left(\frac{1}{\alpha F r^{2}} D(h) \partial_{x} h\right) G^{\prime}(h) d x=0 .
$$

Integrating by parts the last two terms, knowing that $G^{\prime \prime}=\frac{1}{F}$, we get

$$
\frac{d}{d t} \int_{\Omega} G(h) d x+\int_{\Omega} \frac{\left(\partial_{x}^{2} h\right)^{2}}{\alpha W e}+\frac{1}{\alpha F r^{2}} \frac{D(h)}{F(h)}\left(\partial_{x} h\right)^{2} d x=0 .
$$

Finally, integrate in time, we get for all $t \in(0, T)$

$$
\int_{\Omega} G(h(x, t)) d x+\int_{0}^{t} \int_{\Omega} \frac{\left(\partial_{x}^{2} h\right)^{2}}{\alpha W e}+\frac{1}{\alpha F r^{2}} \frac{D(h)}{F(h)}\left(\partial_{x} h\right)^{2} d x d t=\int_{\Omega} G\left(h_{0}(x)\right) d x .
$$

On the other hand, viscous shallow water type models with a general representation of the surface tension and drag term reads

$$
\begin{aligned}
& \partial_{t} h_{\varepsilon}+\partial_{x}\left(h_{\varepsilon} u_{\epsilon}\right)=0, \\
& \partial_{t}\left(h_{\varepsilon} u_{\epsilon}\right)+\partial_{x}\left(h_{\varepsilon} u_{\epsilon}^{2}\right)+\frac{1}{\varepsilon F r^{2}} S\left(h_{\varepsilon}\right) \partial_{x}\left(h_{\varepsilon}\right)=\frac{4}{R_{e}} \partial_{x}\left(h_{\varepsilon} \partial_{x} u_{\epsilon}\right)+\frac{1}{\varepsilon W_{e}} h_{\varepsilon} \partial_{x}^{3} h_{\varepsilon}-\alpha \frac{h_{\varepsilon}^{2}}{\varepsilon T\left(h_{\varepsilon}\right)} u_{\epsilon}
\end{aligned}
$$

with the initial conditions

$$
\left.h_{\varepsilon}\right|_{t=0}=h_{0}^{\varepsilon},\left.\quad\left(h_{\varepsilon} u_{\epsilon}\right)\right|_{t=0}=m_{0}^{\varepsilon}
$$

where $\alpha$ is a positive drag coefficient, $R_{e}, W_{e}$ and $F r$ are respectively the adimentional Reynold, Weber and Froude numbers and $\varepsilon$ is an non-dimensional number. Note that the terms on the right-hand side of the momentum equation represent respectively the viscous term, the capillarity term and the drag term and $\mathrm{S}(\mathrm{h})$ and $\mathrm{T}(\mathrm{h})$ are two nonnegative functions. As an indispensable step to prove global existence of a weak solution of such systems (3), we recall now the BD entropy inequality which was discovered by D. Bresch and B. Desjardins in solving diffusive capillary models of Korteweg type, where they showed that such a non-trivial entropy may govern additional information on the gradient of the density leading to extra regularity on this latter as well as a control of the degenerate term near vacuum, see 7 . So, deriving the mass equation in space, multiplying by $\frac{4}{R_{e}}$, then summing with the momentum equation, and multiplying the sum by the augmented velocity $v_{\varepsilon}=u_{\epsilon}+\frac{4}{R_{e}} \partial_{x}\left(\log h_{\varepsilon}\right)$, the authors obained the following equation:

$$
\begin{aligned}
& \frac{1}{2} \int_{\Omega} \partial_{t}\left(h_{\varepsilon} v_{\varepsilon}^{2}+\frac{1}{\varepsilon W_{e}}\left(\partial_{x} h_{\varepsilon}\right)^{2}\right) d x+\int_{\Omega} \frac{1}{\varepsilon F r^{2}} \partial_{x} h_{\varepsilon} \frac{S\left(h_{\varepsilon}\right)}{h_{\varepsilon}} h_{\varepsilon} u_{\epsilon}+\frac{4 \alpha}{\varepsilon R_{e}} h_{\varepsilon} u_{\epsilon} \frac{\partial_{x} h_{\varepsilon}}{T\left(h_{\varepsilon}\right)} d x \\
& +\int_{\Omega} \frac{4}{R_{e}} \frac{1}{\varepsilon F r^{2}} \frac{S\left(h_{\varepsilon}\right)}{h_{\varepsilon}}\left(\partial_{x} h_{\varepsilon}\right)^{2}+\frac{4}{R_{e}} \frac{1}{\varepsilon W_{e}}\left(\partial_{x}^{2} h_{\varepsilon}\right)^{2}+\alpha \frac{h_{\varepsilon}^{2} u_{\epsilon}^{2}}{\varepsilon T\left(h_{\varepsilon}\right)} d x
\end{aligned}
$$


Indeed, introducing the two functions $T_{0}$ and $S_{0}$ such that

$$
\frac{\partial_{x} h_{\varepsilon}}{T\left(h_{\varepsilon}\right)}=\partial_{x}\left(T_{0}\left(h_{\varepsilon}\right)\right) \quad \text { and } \quad \partial_{x} h_{\varepsilon} \frac{S\left(h_{\varepsilon}\right)}{h_{\varepsilon}}=\partial_{x}\left(S_{0}\left(h_{\varepsilon}\right)\right)
$$

and using the mass equation, the second integral in (4) becomes

$$
\int_{\Omega} \frac{1}{\varepsilon F r^{2}} \partial_{x} h_{\varepsilon} \frac{S\left(h_{\varepsilon}\right)}{h_{\varepsilon}} h_{\varepsilon} u_{\epsilon}+\frac{4 \alpha}{\varepsilon R_{e}} h_{\varepsilon} u_{\epsilon} \frac{\partial_{x} h_{\varepsilon}}{T\left(h_{\varepsilon}\right)} d x=\int_{\Omega} \frac{1}{\varepsilon F r^{2}} \partial_{t} h_{\varepsilon} S_{0}\left(h_{\varepsilon}\right)+\frac{4 \alpha}{\varepsilon R_{e}} \partial_{t} h_{\varepsilon} T_{0}\left(h_{\varepsilon}\right) d x .
$$

Assuming furthermore that there exists two functions $T_{1}$ and $S_{1}$ such that

$$
\partial_{t} h_{\varepsilon} S_{0}\left(h_{\varepsilon}\right)=\partial_{t}\left(S_{1}\left(h_{\varepsilon}\right)\right) \quad \text { and } \quad \partial_{t} h_{\varepsilon} T_{0}\left(h_{\varepsilon}\right)=\partial_{t}\left(T_{1}\left(h_{\varepsilon}\right)\right),
$$

the second integral in 4 yields

$$
\int_{\Omega} \frac{1}{\varepsilon F r^{2}} \partial_{x} h_{\varepsilon} \frac{S\left(h_{\varepsilon}\right)}{h_{\varepsilon}} h_{\varepsilon} u_{\epsilon}+\frac{4 \alpha}{\varepsilon R_{e}} h_{\varepsilon} u_{\epsilon} \frac{\partial_{x} h_{\varepsilon}}{T\left(h_{\varepsilon}\right)} d x=\int_{\Omega} \partial_{t}\left(\frac{1}{\varepsilon F r^{2}} S_{1}\left(h_{\varepsilon}\right)+\frac{4 \alpha}{\varepsilon R_{e}} T_{1}\left(h_{\varepsilon}\right)\right) d x .
$$

Thus, the BD-entropy for 3 reads, for all $t \in(0, T)$

$$
\begin{aligned}
& \int_{\Omega} \frac{1}{2} h_{\varepsilon}(x, t) v_{\varepsilon}(x, t)^{2}+\frac{1}{2} \frac{1}{\varepsilon W_{e}}\left(\partial_{x} h_{\varepsilon}(x, t)\right)^{2}+\frac{1}{\varepsilon F r^{2}} S_{1}\left(h_{\varepsilon}(x, t)\right)+\frac{4 \alpha}{\varepsilon R_{e}} T_{1}\left(h_{\varepsilon}(x, t)\right) d x \\
& -\int_{0}^{t} \int_{\Omega}\left(\frac{4}{R_{e}} \frac{1}{\varepsilon F r^{2}} S\left(h_{\varepsilon}\right)\left(\partial_{x} h_{\varepsilon}\right)^{2}+\frac{4}{R_{e}} \frac{1}{\varepsilon W_{e}}\left(\partial_{x}^{2} h_{\varepsilon}\right)^{2}+\alpha \frac{h_{\varepsilon}^{2} u_{\epsilon}^{2}}{\varepsilon T\left(h_{\varepsilon}\right)}\right) d x d t \\
& =\int_{\Omega} \frac{1}{2} \frac{\left(m_{0}^{\varepsilon}\right)^{2}}{h_{0}^{\varepsilon}}+\frac{1}{2} \frac{1}{\varepsilon W_{e}}\left(\partial_{x} h_{0}^{\varepsilon}(x)\right)^{2}+\frac{1}{\varepsilon F r^{2}} S_{1}\left(h_{0}^{\varepsilon}(x)\right)+\frac{4 \alpha}{\varepsilon R_{e}} T_{1}\left(h_{0}^{\varepsilon}(x)\right) d x .
\end{aligned}
$$

In this paper, as indicated in [5], the main objective is to exhibit a different way to prove the existence of a non-negative global weak solution for (1). In fact, the authors in [1] proved that a nonnegative weak solution of the lubrication model of the form

$$
\partial_{t} h+\partial_{x}\left(F(h) \partial_{x}^{3} h\right)=0 \text { in }(0, T) \times \Omega,\left.\quad h\right|_{t=0}=h_{0} \geq 0 \text { in } \Omega
$$

with $F(h)=h^{n}$ (with $n \geq 1$ ) satisfies the weak formulation of the above system, as well as the BF dissipative entropy given in (2). This has been generalized in several papers as mentionned previously. On the other hand, a weak solution of system (3) would satisfy its corresponding weak formulation as well as the energy and BD-entropy inequality: see for instance [7] and more recently [21], [19], [11] and the recent handbook [9]. The key tool in our method is to prove an existence result for system (1) by passing the limit of $\varepsilon$ to zero in both the weak formulation of (3) as well as the BD-entropy. One would readily infer that, more precisely, we will prove that the BD entropy of the appropriate viscous shallow water system will implies the different BF dissipative entropy of the lubrication system at the limit playing with the drag terms and the capillarity forces. Different BF entropies are obtained depending on the choice of drag terms generalizing in some sense papers such as 18, 14.

For readers convenience, we decompose the paper in three sections. In the first section we will discuss the case $S(r)=r$ and $T(r)=F(r)=r^{2}+r^{3}$ which corresponds to the viscous shallow water with drag term formally derived in 15] and mathematically justified in [10. Then we discuss a viscous compressible system with a nonlinear drag term and the link with some results that we can find for instance in [2], 3], 12] and [16]. Finally we conclude with more general capillarity terms with no pressure on the viscous compressible system to derive general forms of fourth-order equation including the Derrida-Lebowitz-Speer-Spohn equation. 


\section{The limit of A Viscous Shallow WATER MOdel FORMAlly DERIVED IN [15] AND JUSTIFIED IN 10}

In this section, we will consider the viscous shallow water model given in $(3)$ regarding that $S(r)=r$ and $T(r)=F(r)=r^{2}+r^{3}$. This choice corresponds to the model formally derived in 15 and mathematically justified in [10]. We will prove that it will be linked to the lubrication model studied for instance by A.L. Bertozzi and M. Pugh. More precisely, consider the Shallow Water system corresponding to the new drag term $\frac{h_{\varepsilon}^{2}}{F\left(h_{\varepsilon}\right)} u_{\epsilon}$, defined in a periodic domain $\Omega$ :

$$
\begin{aligned}
& \partial_{t} h_{\varepsilon}+\partial_{x}\left(h_{\varepsilon} u_{\epsilon}\right)=0, \\
& \varepsilon\left(\partial_{t}\left(h_{\varepsilon} u_{\epsilon}\right)+\partial_{x}\left(h_{\varepsilon} u_{\epsilon}^{2}\right)\right)+\frac{h_{\varepsilon} \partial_{x}\left(h_{\varepsilon}\right)}{\mathrm{Fr}^{2}}=\varepsilon\left(\frac{4}{\mathrm{R}_{\mathrm{e}}} \partial_{x}\left(h_{\varepsilon} \partial_{x} u_{\epsilon}\right)\right)+\frac{1}{\mathrm{~W}_{\mathrm{e}}} h_{\varepsilon} \partial_{x}^{3} h_{\varepsilon}-\alpha \frac{u_{\epsilon}}{1+h_{\varepsilon}} .
\end{aligned}
$$

The initial conditions are given by :

$$
\left.h_{\varepsilon}\right|_{t=0}=h_{0}^{\varepsilon},\left.\quad\left(h_{\varepsilon} u_{\epsilon}\right)\right|_{t=0}=m_{0}^{\varepsilon} .
$$

\subsection{Formal limit}

Assuming enough uniform boundedness on the different terms of the above system, and taking $\varepsilon \rightarrow 0$ in (9), we obtain

$$
\begin{aligned}
& \partial_{t} h+\partial_{x}(h u)=0, \\
& h u=\frac{1}{\alpha W_{e}} F(h) \partial_{x}^{3} h-\frac{1}{\alpha F r^{2}} F(h) \partial_{x} h,
\end{aligned}
$$

with $F(h)=h^{2}+h^{3}$ which can be written equivalently

$$
\partial_{t} h+\partial_{x}\left(\frac{1}{\alpha W_{e}} F(h)\right) \partial_{x}^{3} h-\frac{1}{\alpha F r^{2}} F(h) \partial_{x} h=0 .
$$

The mathematical justification of this model relies on an energy method with an indispensable contribution of the BD-entropy.

\subsection{Mathematical justification}

The first essential step herein is the a priori estimate which is maintained by the uniform bounds that both the energy and BD-entropy offer. The energy equation corresponding to 9 is obtained by multiplying the momentum equation in (1) by $u_{\varepsilon}$. Integrating then in space and using the mass equation, we obtain:

$$
\frac{d}{d t}\left(\int_{\Omega} \varepsilon \frac{h_{\varepsilon} u_{\epsilon}^{2}}{2}+\frac{h_{\varepsilon}^{2}}{2 F r^{2}}+\frac{\left(\partial_{x} h_{\varepsilon}\right)^{2}}{2 W_{e}}\right) d x+\int_{\Omega} \frac{4 \varepsilon}{R_{e}} h_{\varepsilon}\left(\partial_{x} u_{\epsilon}\right)^{2}+\alpha \frac{h_{\varepsilon}^{2} u_{\epsilon}^{2}}{F\left(h_{\varepsilon}\right)} d x=0 .
$$

As for the BD-entropy inequality, it reads

$$
\begin{aligned}
& \frac{1}{2} \frac{d}{d t} \int_{\Omega} h_{\varepsilon} v_{\varepsilon}^{2} d x+\frac{1}{\varepsilon}\left[\frac{1}{2} \frac{d}{d t} \int_{\Omega} \frac{h_{\varepsilon}^{2}}{F r^{2}}+\frac{\left(\partial_{x} h_{\varepsilon}\right)^{2}}{W_{e}} d x+\alpha \int_{\Omega} \frac{h_{\varepsilon}^{2} u_{\epsilon}^{2}}{F\left(h_{\varepsilon}\right)} d x\right] \\
& +\frac{4}{\varepsilon \mathrm{R}_{\mathrm{e}}}\left[\int_{\Omega} \frac{\left(\partial_{x} h_{\varepsilon}\right)^{2}}{\mathrm{Fr}^{2}} d x+\int_{\Omega} \frac{\left(\partial_{x}^{2} h_{\varepsilon}\right)^{2}}{\mathrm{~W}_{\mathrm{e}}} d x+\alpha \int_{\Omega} \frac{h_{\varepsilon} u_{\epsilon}}{F\left(h_{\varepsilon}\right)} \partial_{x} h_{\varepsilon} d x\right]=0,
\end{aligned}
$$


where $v_{\varepsilon}=u_{\epsilon}+\frac{4 \alpha}{R_{e}} \partial_{x}\left(\log h_{\varepsilon}\right)$.

The last term can be rewritten as:

$$
\begin{aligned}
\int_{\Omega} \frac{h_{\varepsilon} u_{\epsilon}}{F\left(h_{\varepsilon}\right)} \partial_{x} h_{\varepsilon} & =\int_{\Omega} h_{\varepsilon} u_{\epsilon} \frac{\partial_{x} h_{\varepsilon}}{h_{\varepsilon}^{2}+h_{\varepsilon}^{3}} \\
& =\int_{\Omega} h_{\varepsilon} u_{\epsilon} \partial_{x} h_{\varepsilon}\left(\frac{1}{h_{\varepsilon}^{2}}+\frac{1}{1+h_{\varepsilon}}-\frac{1}{h_{\varepsilon}}\right) \\
& =\int_{\Omega} h_{\varepsilon} u_{\epsilon} \partial_{x}\left(\frac{-1}{h_{\varepsilon}}+\log \left(1+h_{\varepsilon}\right)-\log \left(h_{\varepsilon}\right)\right) \\
& =\frac{d}{d t} \int_{\Omega} \underbrace{-\left(1+h_{\varepsilon}\right) \log \left(\frac{h_{\varepsilon}}{1+h_{\varepsilon}}\right)}_{>0} .
\end{aligned}
$$

Finally, the BD-entropy reads

$$
\begin{aligned}
& \frac{d}{d t} \int_{\Omega} \frac{\varepsilon}{2} h_{\varepsilon} v_{\varepsilon}^{2}-\frac{4 \alpha}{R_{e}}\left(1+h_{\varepsilon}\right) \log \left(\frac{h_{\varepsilon}}{1+h_{\varepsilon}}\right)+\frac{h_{\varepsilon}^{2}}{2 F r^{2}}+\frac{\left(\partial_{x} h_{\varepsilon}\right)^{2}}{2 W_{e}} d x \\
& +\alpha \int_{\Omega} \frac{h_{\varepsilon}^{2} u_{\epsilon}^{2}}{F\left(h_{\varepsilon}\right)}+\frac{4}{\mathrm{R}_{\mathrm{e}}}\left[\int_{\Omega} \frac{\left(\partial_{x} h_{\varepsilon}\right)^{2}}{\mathrm{Fr}^{2}} d x+\frac{\left(\partial_{x x} h_{\varepsilon}\right)^{2}}{\mathrm{~W}_{\mathrm{e}}} d x\right]=0 .
\end{aligned}
$$

Let's recall the definition of the weak formulation of system (9):

Definition 1.1. A weak formulation of the shallow water model with the nonlinear drag term represented in system [9] is given by

$$
\int_{0}^{\infty} \int_{\Omega} h_{\varepsilon} \partial_{t} \psi d x d t+\int_{\Omega} h_{0}^{\varepsilon} \psi(\cdot, 0) d x=-\int_{0}^{\infty} \int_{\Omega} h_{\varepsilon} u_{\epsilon} \partial_{x} \psi d x d t
$$

and

$$
\begin{aligned}
& \varepsilon\left(\int_{0}^{\infty} \int_{\Omega} h_{\varepsilon} u_{\epsilon} \partial_{t} \phi d x d t+\int_{\Omega} m_{0}^{\varepsilon} \phi(\cdot, 0) d x+\int_{0}^{\infty} \int_{\Omega} h_{\varepsilon} u_{\epsilon}^{2} \partial_{x} \phi d x d t\right) \\
& -\frac{4 \varepsilon}{R_{e}} \int_{0}^{\infty} \int_{\Omega} h_{\varepsilon} \partial_{x} u_{\epsilon} \partial_{x} \phi d x d t+\frac{1}{W_{e}} \int_{0}^{\infty} \int_{\Omega} \frac{1}{2}\left(\partial_{x} h_{\varepsilon}\right)^{2} \partial_{x} \phi d x d t \\
& -\frac{1}{W_{e}} \int_{0}^{\infty} \int_{\Omega} h_{\varepsilon} \partial_{x}^{2} h_{\varepsilon} \partial_{x} \phi d x d t+\frac{1}{F r^{2}} \int_{0}^{\infty} \int_{\Omega} h_{\varepsilon}^{2} \partial_{x} \phi d x d t-\alpha \int_{0}^{\infty} \int_{\Omega} \frac{h_{\varepsilon}^{2} u_{\epsilon}}{F\left(h_{\varepsilon}\right)} \phi d x d t=0,
\end{aligned}
$$

for all $\psi \in \mathcal{C}_{0}^{\infty}(\Omega \times[0, \infty))$ and $\phi \in \mathcal{C}_{0}^{\infty}(\Omega \times[0, \infty))$.

To proceed, we give first the definition of a global weak solution of (9), as well as its existence result which follows the proof in 7

Definition 1.2. A couple $(h, u)$ is said to be a global weak solution of 9 if it satisfies the weak formulations (14)-15) as well as the energy and BD-entropy inequalities respectively given by

$\sup _{t \in(0, T)}\left(\int_{\Omega} \varepsilon \frac{h_{\varepsilon} u_{\epsilon}^{2}}{2}+\frac{h_{\varepsilon}^{2}}{2 F r^{2}}+\frac{\left(\partial_{x} h_{\varepsilon}\right)^{2}}{2 W_{e}} d x\right)(t)+\int_{0}^{T} \int_{\Omega} \frac{4 \varepsilon}{R_{e}} h_{\varepsilon}\left(\partial_{x} u_{\epsilon}\right)^{2}+\alpha \frac{h_{\varepsilon}^{2} u_{\epsilon}^{2}}{F\left(h_{\varepsilon}\right)} d x \leq\left(\int_{\Omega} \varepsilon \frac{m_{0}^{\varepsilon}}{2 h_{0}^{\varepsilon}}+\frac{\left(h_{0}^{\varepsilon}\right)^{2}}{2 F r^{2}}+\frac{\left(\partial_{x} h_{0}^{\varepsilon}\right)^{2}}{2 W_{e}}\right) d x$ 
and

$$
\begin{aligned}
& \sup _{t \in(0, T)}\left(\int_{\Omega} \frac{\varepsilon}{2} h_{\varepsilon} v_{\varepsilon}^{2}-\frac{4 \alpha}{R_{e}}\left(1+h_{\varepsilon}\right) \log \left(\frac{h_{\varepsilon}}{1+h_{\varepsilon}}\right)+\frac{h_{\varepsilon}^{2}}{2 F r^{2}}+\frac{\left(\partial_{x} h_{\varepsilon}\right)^{2}}{2 W_{e}} d x\right)(t) \\
& +\alpha \int_{0}^{T} \int_{\Omega} \frac{h_{\varepsilon}^{2} u_{\epsilon}^{2}}{F\left(h_{\varepsilon}\right)}+\frac{4}{\mathrm{R}_{\mathrm{e}}}\left[\int_{0}^{T} \int_{\Omega} \frac{\left(\partial_{x} h_{\varepsilon}\right)^{2}}{\mathrm{Fr}^{2}} d x+\int_{0}^{T} \int_{\Omega} \frac{\left(\partial_{x x} h_{\varepsilon}\right)^{2}}{\mathrm{~W}_{\mathrm{e}}} d x\right] \\
& \leq \int_{\Omega} \frac{\varepsilon}{2}\left(\frac{m_{0}^{\varepsilon}}{\rho_{0}^{\varepsilon}}\right)^{2}-\frac{4 \alpha}{R_{e}}\left(1+h_{0}^{\varepsilon}\right) \log \left(\frac{h_{0}^{\varepsilon}}{1+h_{0}^{\varepsilon}}\right)+\frac{\left(h_{0}^{\varepsilon}\right)^{2}}{2 F r^{2}}+\frac{\left(\partial_{x} h_{0}^{\varepsilon}\right)^{2}}{2 W_{e}} d x
\end{aligned}
$$

The existing result of global weak solutions reads

Theorem 1.1. Let $\left(h_{0}^{\varepsilon}, m_{0}^{\varepsilon}\right)$ be such that $h_{0}^{\varepsilon}>0$ and

$$
\begin{gathered}
h_{0}^{\varepsilon} \in H^{1}(\Omega), \quad \varepsilon\left|m_{0}^{\varepsilon}\right|^{2} / h_{0}^{\varepsilon} \in L^{1}(\Omega), \quad \sqrt{\varepsilon} \partial_{x} \sqrt{h_{0}^{\varepsilon}} \in L^{2}(\Omega), \\
-\left(1+h_{0}^{\varepsilon}\right) \log \left(\frac{h_{0}^{\varepsilon}}{1+h_{0}^{\varepsilon}}\right) \in L^{1}(\Omega),
\end{gathered}
$$

then there exists a global weak solution of (9) in the sense of definition 1.1.

Note that we do not give details on the existence of global weak solutions to the viscous-shallow water system and refer the interested reader to 9 where the authors explain the procedures in details. Remark that the new quantities here concern drag terms which are compatible with the mathematical procedure.

The main result in this part is the following result

Theorem 1.2. Given a sequence $\left(h_{\varepsilon}, u_{\epsilon}\right)_{\varepsilon}$ a global solution of $(9)$, satisfying the initial conditions given in the previous theorem, in the sense of Theorem 1.1 then there exists a subsequence of $\left(h_{\varepsilon}, u_{\epsilon}\right)$ that converges to a couple $(h, u)$, which is a solution of the weak formulation of the lubrication system satisfying the initial condition $\left.h\right|_{t=0}=h_{0}$, where $h_{0}$ is the weak limit of $h_{0}^{\varepsilon}$ in $H^{1}(\Omega)$.

Proof. The proof starts from integrating the energy and BD-energy inequalities in the time interval $(0, \mathrm{t})$, for all $\mathrm{t}$ in $(0, \mathrm{~T})$. We obtain

$$
\begin{aligned}
& \frac{\varepsilon}{2} \int_{\Omega} h_{\varepsilon}(x, t) u_{\epsilon}(x, t)^{2} d x+\frac{1}{2 F r^{2}} \int_{\Omega} h_{\varepsilon}(x, t)^{2} d x+\frac{1}{2 W_{e}} \int_{\Omega}\left(\partial_{x} h_{\varepsilon}(x, t)\right)^{2} d x \\
& +\frac{4 \varepsilon}{R_{e}} \int_{0}^{t} \int_{\Omega} h_{\varepsilon}\left(\partial_{x} u_{\epsilon}\right)^{2} d x d t+\alpha \int_{0}^{t} \int_{\Omega} \frac{h_{\varepsilon}^{2} u_{\epsilon}^{2}}{F\left(h_{\varepsilon}\right)} d x d t \leq \frac{1}{2} \int_{\Omega} \frac{\left(m_{0}^{\varepsilon}(x)\right)^{2}}{h_{0}^{\varepsilon}(x)}+\frac{h_{0}^{\varepsilon}(x)^{2}}{F r^{2}}+\frac{\left(\partial_{x} h_{0}^{\varepsilon}(x)\right)^{2}}{W_{e}} d x,
\end{aligned}
$$

and

$$
\begin{aligned}
& \frac{\varepsilon}{2} \int_{\Omega} h_{\varepsilon}(x, t) v_{\varepsilon}(x, t)^{2} d x+\frac{1}{2 F r^{2}} \int_{\Omega} h_{\varepsilon}(x, t)^{2} d x+\frac{1}{2 W_{e}} \int_{\Omega}\left(\partial_{x} h_{\varepsilon}(x, t)\right)^{2} d x-\frac{4 \alpha}{R_{e}} \int_{\Omega}\left(1+h_{\varepsilon}(x, t)\right) \log \left(\frac{h_{\varepsilon}(x, t)}{1+h_{\varepsilon}(x, t)}\right) d x \\
& +\alpha \int_{0}^{t} \int_{\Omega} \frac{h_{\varepsilon}^{2} u_{\epsilon}^{2}}{F\left(h_{\varepsilon}\right)} d x d t+\frac{4}{R_{e}} \int_{0}^{t} \int_{\Omega} \frac{\left(\partial_{x} h_{\varepsilon}\right)^{2}}{F r^{2}}+\frac{\left(\partial_{x}^{2} h_{\varepsilon}\right)^{2}}{W_{e}} d x d t \\
& \leq \frac{\varepsilon}{2} \int_{\Omega}\left(\frac{\left(m_{0}^{\varepsilon}(x)\right)^{2}}{h_{0}^{\varepsilon}(x)}+2 \frac{m_{0}^{\varepsilon}(x)}{h_{0}^{\varepsilon}(x)} \partial_{x} h_{0}^{\varepsilon}(x)+\frac{\left(\partial_{x} h_{0}^{\varepsilon}(x)\right)^{2}}{h_{0}^{\varepsilon}(x)}\right) d x+\int_{\Omega} \frac{\left(h_{0}^{\varepsilon}(x)\right)^{2}}{2 F r^{2}} d x+\int_{\Omega} \frac{\left(\partial_{x} h_{0}^{\varepsilon}(x)\right)^{2}}{2 W_{e}} d x \\
& -\frac{4 \alpha}{R_{e}} \int_{\Omega}\left(1+h_{0}^{\varepsilon}(x)\right) \log \left(\frac{h_{0}^{\varepsilon}(x)}{1+h_{0}^{\varepsilon}(x)}\right) d x
\end{aligned}
$$


Using the uniform bounds on the initial data introduced in theorem 1.1, and since $h_{0}^{\varepsilon}$ is bounded, we obtain that the right hand side of each of the above equalities is bounded uniformly in $\varepsilon$. Thus, the energy yields the following estimates

$$
\begin{gathered}
\sqrt{\varepsilon}\left\|\sqrt{h}_{\varepsilon} u_{\epsilon}\right\|_{L^{\infty}\left(0, T ; L^{2}(\Omega)\right)} \leq C, \\
\left\|h_{\varepsilon}\right\|_{L^{\infty}\left(0, T ; H^{1}(\Omega)\right)} \leq C, \\
\sqrt{\varepsilon}\left\|\sqrt{h}_{\varepsilon} \partial_{x} u_{\epsilon}\right\|_{L^{2}\left(0, T ; L^{2}(\Omega)\right)} \leq C, \\
\left\|\frac{u_{\epsilon}}{\sqrt{1+h_{\varepsilon}}}\right\|_{L^{2}\left(0, T ; L^{2}(\Omega)\right)} \leq C .
\end{gathered}
$$

From (21), and applying Rellich-Kandrachov compactness theory (since $\Omega$ is bounded and Lipschitz domain), we get that $H^{1}(\Omega) \subset L^{\infty}(\Omega)$, and thus

$$
\left\|h_{\varepsilon}\right\|_{L^{\infty}(0, T, \Omega)} \leq\left\|h_{\varepsilon}\right\|_{L^{\infty}\left(0, T, H^{1}(\Omega)\right)} \leq C .
$$

A further look on the fifth term in (18) allows to get a uniform bound on $u_{\epsilon}$ in $L^{2}\left(0, T ; L^{2}(\Omega)\right)$. Indeed

$$
\begin{aligned}
\int_{0}^{t} \int_{\Omega} u_{\epsilon}^{2} d x d t & =\int_{0}^{t} \int_{\Omega} \frac{\sqrt{\left(1+h_{\varepsilon}\right)}}{\sqrt{\left(1+h_{\varepsilon}\right)}} u_{\epsilon}^{2} d x d t \\
& =\int_{0}^{t} \int_{\Omega} \frac{\eta}{2}\left(1+h_{\varepsilon}\right) u_{\epsilon}^{2}+\frac{1}{2 \eta} \frac{u_{\epsilon}^{2}}{1+h_{\varepsilon}} d x d t \\
& \leq \frac{\eta}{2}(1+M) \int_{0}^{t} \int_{\Omega} u_{\epsilon}^{2} d x d t+C,
\end{aligned}
$$

where $\mathrm{M}$ is chosen due to Sobolev injections and the uniform boundedness of $u_{\epsilon}$ in $L^{\infty}\left(0, T ; H^{1}(\Omega)\right)$ such that

$$
\left\|h_{\varepsilon}\right\|_{L^{\infty}(0, T, \Omega)} \leq M
$$

Therefore, choosing $\eta$ such that $\eta \leq \frac{1}{1+M}$, we obtain

$$
\left\|u_{\epsilon}\right\|_{L^{2}\left(0, T ; L^{2}(\Omega)\right)} \leq C
$$

We can deduce as well that

$$
\int_{0}^{t} \int_{\Omega}\left(\frac{u_{\epsilon}}{1+h_{\varepsilon}}\right)^{2} d x d t \leq \int_{0}^{t} \int_{\Omega} u_{\epsilon}^{2} d x d t \leq C .
$$

As for the additional estimates provided by the BD-entropy, we get

$$
\begin{gathered}
\sqrt{\varepsilon}\left\|\partial_{x}\left(\sqrt{h}_{\varepsilon}\right)\right\|_{L^{\infty}\left(0, T, L^{2}(\Omega)\right)} \leq C, \\
\left\|-\left(1+h_{\varepsilon}\right) \log \left(\frac{h_{\varepsilon}}{1+h_{\varepsilon}}\right)\right\|_{L^{\infty}\left(0, T, L^{1}(\Omega)\right)} \leq C, \\
\left\|\partial_{x} h_{\varepsilon}\right\|_{L^{2}\left(0, T, H^{1}(\Omega)\right)} \leq C \quad \text { and }\left\|\partial_{x} h_{\varepsilon}\right\|_{H^{1}((0, T) \times \Omega)} \leq C .
\end{gathered}
$$

Having such bounds, on can deduce some consequences, mainly using (21) and (25), such as

$$
\begin{gathered}
\partial_{x}\left(h_{\varepsilon} u_{\epsilon}\right) \text { bounded uniformly in } L^{2}\left(0, T, H^{-1}(\Omega)\right), \\
h_{\varepsilon} u_{\epsilon}, h_{\varepsilon} \partial_{x}^{2} h_{\varepsilon} \text { bounded uniformly in } L^{2}\left(0, T, L^{2}(\Omega)\right),
\end{gathered}
$$

Now we utilize the various uniform bounds obtained on the different unknowns of the system to conclude some convergence results. Starting from (25), we can infer that there exists $u$ in $L^{2}\left(0, T, L^{2}(\Omega)\right)$ such that, up to a subsequence, $u_{\epsilon}$ converges weakly to $u$ in $L^{2}\left(0, T, L^{2}(\Omega)\right)$. Since $\Omega$ is a bounded Lipschitz domain, then 
applying compactness theory since $H^{1}(\Omega)$ is compactly injected in $L^{2}(\Omega)$. But having $h_{\varepsilon}$ bounded uniformly in $L^{\infty}\left(0, T, H^{1}(\Omega)\right)$, and $\partial_{t} h_{\varepsilon}=-\partial_{x}\left(h_{\varepsilon} u_{\epsilon}\right)$ bounded uniformly in $L^{2}\left(0, T, H^{-1}\right)$, by Aubin-Simon's theorem we find that $h_{\varepsilon}$ converges strongly to some $h$ in $C\left([0, T], L^{2}(\Omega)\right)$. Also, due to the uniform boundedness of $\partial_{x} h_{\varepsilon}$ in $H^{1}((0, T) \times \Omega)$, we get that $h_{\varepsilon}$ is in $L^{2}\left(0, T, H^{2}(\Omega)\right)$. But since the embedding of $H^{2}(\Omega)$ in $H^{1}(\Omega)$ is compact, again using Aubin-Simon's theorem we get that $h_{\varepsilon}$ also converges strongly to $h$ in $L^{2}\left(0, T, H^{1}(\Omega)\right)$. In fact, one consequence of these latter results is that

$$
h_{\varepsilon}(x, 0) \rightarrow h(x, 0) \quad \text { in } \quad H^{1}(\Omega) .
$$

We denote by $h_{0}$ the weak limit in $H^{1}(\Omega)$ of $h_{0}^{\varepsilon}$. Finally, passing to the limit in the weak formulation (14)-(15) of system (9), we obtain:

(1) using uniform boundedness of $\partial_{x} h_{\varepsilon}$ in $H^{1}((0, T) \times \Omega)$, we get the strong convergence and thus weak convergence of $h_{\varepsilon}$ to $h$ in $L^{2}((0, T) \times \Omega)$. Thus we have that

$$
\int_{0}^{t} \int_{\Omega} h_{\varepsilon} \partial_{t} \psi d x d t \underset{\varepsilon \longrightarrow 0}{\longrightarrow} \int_{0}^{t} \int_{\Omega} h \partial_{t} \psi d x d t .
$$

(2) $h_{\varepsilon}$ converges to $h$ weakly in $C\left(0, T, L^{2}(\Omega)\right)$, hence

$$
\int_{\Omega} h_{0}^{\varepsilon} \psi(., 0) d x \underset{\varepsilon \longrightarrow 0}{\longrightarrow} \int_{\Omega} h_{0} \psi(., 0) d x .
$$

(3) Due to strong convergence of $h_{\varepsilon}$ in $L^{2}((0, T) \times \Omega)$, and the boundedness and weak convergence of $u_{\epsilon}$ in $L^{2}\left(0, T, L^{2}(\Omega)\right)$, we get that $h_{\varepsilon} u_{\epsilon}$ connverges weakly to $h u$ in $L^{2}\left(0, T, L^{2}(\Omega)\right)$

$$
\begin{aligned}
\int_{0}^{t} \int_{\Omega}\left(h_{\varepsilon} u_{\epsilon}-h u\right) \partial_{x} \psi d x d t & =\int_{0}^{t} \int_{\Omega}\left(h_{\varepsilon}-h\right) u_{\epsilon} \partial_{x} \psi d x d t+\int_{0}^{t} \int_{\Omega}\left(u_{\epsilon}-u\right) h \partial_{x} \psi d x d t \\
& \leq\left\|\partial_{x} \psi\right\|_{L^{\infty}((0, T) \times \Omega)}\left\|u_{\epsilon}\right\|_{L^{2}\left(0, T ; L^{2}(\Omega)\right)}\left\|h_{\varepsilon}-h\right\|_{L^{2}\left(0, T ; L^{2}(\Omega)\right)}+\int_{0}^{t} \int_{\Omega}\left(u_{\epsilon}-u\right) h \partial_{x} \psi d x d t \\
& \longrightarrow 0 \\
& \longrightarrow 00
\end{aligned}
$$

(4) We follow now the same analysis for (15): the integrals multiplied by $\varepsilon$ tend to zero upon the limit as they are uniformly bounded

$$
\begin{aligned}
& \varepsilon\left(\int_{0}^{\infty} \int_{\Omega} h_{\varepsilon} u_{\epsilon} \partial_{t} \phi d x d t+\int_{\Omega} m_{0}^{\varepsilon} \phi(\cdot, 0) d x+\int_{0}^{\infty} \int_{\Omega} h_{\varepsilon} u_{\epsilon}^{2} \partial_{x} \phi d x d t-\frac{4}{R_{e}} \int_{0}^{\infty} \int_{\Omega} h_{\varepsilon} \partial_{x} u_{\epsilon} \partial_{x} \phi d x d t\right) \\
& \leq \varepsilon \max \left(\left\|h_{\varepsilon}\right\|_{L^{\infty}((0, T) \times \Omega)},\left\|u_{\epsilon}\right\|_{L^{2}\left(0, T ; L^{2}(\Omega)\right)},\left\|m_{0}^{\varepsilon}\right\|_{L^{2}(\Omega)},\left\|\partial_{t} \phi\right\|_{L^{2}\left(0, T ; L^{2}(\Omega)\right.}\|\phi\|_{L^{\infty}\left(0, T ; L^{2}(\Omega)\right.},\left\|\partial_{x} \phi\right\|_{L^{\infty}((0, T) \times \Omega)}\right) \\
& \quad+\sqrt{\varepsilon}\left\|\sqrt{\varepsilon} \sqrt{h_{\varepsilon}} \partial_{x} u_{\epsilon}\right\|_{L^{2}\left(0, T ; L^{2}(\Omega)\right.} \\
& \leq \varepsilon C_{1}+\sqrt{\varepsilon} C_{2} \underset{\varepsilon \longrightarrow 0}{\longrightarrow} .
\end{aligned}
$$

(5) As the for fifth and sixth terms, we use the results obtained in (21), (31), the strong convergence of $h_{\varepsilon}$ to $h$ in $C\left(0, T, L^{2}(\Omega)\right)$ and $L^{2}\left(0, T, H^{1}(\Omega)\right)$, and the weak convergence of $\partial_{x}^{2} h_{\varepsilon}$ in $L^{2}\left(0, T, L^{2}(\Omega)\right)$, we 
get

$$
\begin{aligned}
& \int_{0}^{t} \int_{\Omega} h_{\varepsilon} \partial_{x}^{2} h_{\varepsilon} \partial_{x} \phi-h \partial_{x}^{2} h \partial_{x} \phi d x d t=\int_{0}^{t} \int_{\Omega}\left(h_{\varepsilon}-h\right) \partial_{x}^{2} h_{\varepsilon} \partial_{x} \phi d x d t+\int_{0}^{t} \int_{\Omega}\left(\partial_{x}^{2} h_{\varepsilon}-\partial_{x}^{2} h\right) h \partial_{x} \phi d x d t \\
& \leq\left\|\partial_{x} \phi\right\|_{\left.L^{\infty}((0, T) \times \Omega)\right)}\left\|\partial_{x}^{2} h_{\varepsilon}\right\|_{L^{2}\left(0, T ; L^{2}(\Omega)\right)}\left\|h_{\varepsilon}-h\right\|_{L^{2}\left(0, T ; L^{2}(\Omega)\right.} \\
& +\int_{0}^{t} \int_{\Omega}\left(\partial_{x}^{2} h_{\varepsilon}-\partial_{x}^{2} h\right) h \partial_{x} \phi d x d t \\
& \longrightarrow 0 \text {. }
\end{aligned}
$$

As for the sixth term, we have deduced that $\partial_{x} h_{\varepsilon}$ converges strongly to $\partial_{x} h$ in $L^{2}\left(0, T ; L^{2}(\Omega)\right.$, thus we get the strong and hence weak convergence of $\left(\partial_{x} h_{\varepsilon}\right)^{2}$ to $\left(\partial_{x} h\right)^{2}$ in $L^{1}((0, T) \times \Omega)$, hence

$$
\int_{0}^{t} \int_{\Omega}\left(\partial_{x} h_{\varepsilon}\right)^{2} \partial_{x} \phi d x d t \underset{\varepsilon \longrightarrow 0}{\longrightarrow} \int_{0}^{t} \int_{\Omega}\left(\partial_{x} h\right)^{2} \partial_{x} \phi d x d t .
$$

(6) As for the last term, we use the fact that due to strong convergence of $h_{\varepsilon}$ to $h$ in $L^{2}((0, T,) \times \Omega)$, and weak convergence of $u_{\epsilon}$ to $u$ in $L^{2}\left(0, T ; L^{2}(\Omega)\right.$ we get

$$
\begin{aligned}
\int_{0}^{t} \int_{\Omega} \frac{u_{\epsilon}}{1+h_{\varepsilon}} \phi-\frac{u}{1+h_{\varepsilon}} \phi d x d t & =\int_{0}^{t} \int_{\Omega} u_{\epsilon} \phi\left(\frac{1}{1+h_{\varepsilon}}-\frac{1}{1+h}\right) d x d t+\int_{0}^{t} \int_{\Omega} \frac{1}{1+h} \phi\left(u_{\epsilon}-u\right) d x d t \\
& \leq\|\phi\|_{\left.L^{\infty}((0, T) \times \Omega)\right)}\left\|u_{\epsilon}\right\|_{L^{2}\left(0, T ; L^{2}(\Omega)\right)} \int_{0}^{t} \int_{\Omega} \frac{\left(h_{\varepsilon}-h\right)^{2}}{(1+h)\left(1+h_{\varepsilon}\right)} d x d t \\
& +\int_{0}^{t} \int_{\Omega} \frac{1}{1+h} \phi\left(u_{\epsilon}-u\right) d x d t \\
& \leq C \int_{0}^{t} \int_{\Omega}\left(h_{\varepsilon}-h\right)^{2} d x d t+\int_{0}^{t} \int_{\Omega} \frac{1}{1+h} \phi\left(u_{\epsilon}-u\right) d x d t \\
& \longrightarrow 0
\end{aligned}
$$

where we have used the fact that $\frac{1}{1+h} \phi \in L^{2}\left(0, T ; L^{2}(\Omega)\right)$.

Hence, we get that the couple $(h, u)$ satisfies

$$
\begin{aligned}
& \int_{0}^{t} \int_{\Omega} h \partial_{t} \psi d x d t+\int_{\Omega} h_{0} \psi(., 0) d x=-\int_{0}^{t} \int_{\Omega} h u \partial_{x} \psi d x d t \\
& \frac{1}{W_{e}} \int_{0}^{\infty} \int_{\Omega} \frac{1}{2}\left(\partial_{x} h\right)^{2} \partial_{x} \phi d x d t-\frac{1}{W_{e}} \int_{0}^{\infty} \int_{\Omega} h \partial_{x}^{2} h \partial_{x} \phi d x d t \\
& +\frac{1}{F r^{2}} \int_{0}^{\infty} \int_{\Omega} h^{2} \partial_{x} \phi d x d t-\alpha \int_{0}^{\infty} \int_{\Omega} \frac{h^{2} u}{F\left(h_{\varepsilon}\right)} \phi d x d t=0
\end{aligned}
$$

which is the weak formulation of the lubrication system.

\subsection{BF-entropy information include in the limit part of the BD-entropy}

In the previous subsections, we have proved that a weak limit of the shallow water system with the chosen nonlinear drag term (9) would converge to a couple $(h, u)$ satisfying the weak formulation of the lubrication system 10. If we can now show that $(h, u)$ satisfies as well the BF dissipative entropy, then certainly we can say that weak solution of shallow water system (9) converges weakly to the weak solution of the lubrication system (11) as defined by 1]. One significant consequence this approach shows is the implicitness of the lubrication 
system in the shallow water system. in particular one notices the inclusion of the BF dissipative entropy in the $\mathrm{BD}$ entropy which explains the convergence limit relation in between. One can naively predict that not just the entropy relations, but also other characteristics of both system are supposed to be interlaced with one another, and that under physical assumptions and bounded initial datum, the lubrication theory originates from shallow water assumptions in the weak sense as well.

The aim now, as mentioned above, is to prove the convergence of the BD entropy into the BF dissipative entropy. Lets recall first each of the BD entropy of system (9), and the BF dissipative entropy of the sought for lubrication model (11). In fact, the BD entropy (17) reads

$$
\begin{aligned}
& \frac{\varepsilon}{2} \int_{\Omega} h_{\varepsilon}(x, t) v_{\varepsilon}(x, t)^{2} d x+\frac{1}{2 F r^{2}} \int_{\Omega} h_{\varepsilon}(x, t)^{2} d x+\frac{1}{2 W_{e}} \int_{\Omega}\left(\partial_{x} h_{\varepsilon}(x, t)\right)^{2} d x-\frac{4 \alpha}{R_{e}} \int_{\Omega}\left(1+h_{\varepsilon}(x, t)\right) \log \left(\frac{h_{\varepsilon}(x, t)}{1+h_{\varepsilon}(x, t)}\right) d x \\
& +\alpha \int_{0}^{t} \int_{\Omega} \frac{h_{\varepsilon}^{2} u_{\epsilon}^{2}}{F\left(h_{\varepsilon}\right)} d x d t+\frac{4}{R_{e}} \int_{0}^{t} \int_{\Omega} \frac{\left(\partial_{x} h_{\varepsilon}\right)^{2}}{F r^{2}}+\frac{\left(\partial_{x}^{2} h_{\varepsilon}\right)^{2}}{W_{e}} d x d t \\
& \leq \frac{\varepsilon}{2} \int_{\Omega}\left(\frac{\left(m_{0}^{\varepsilon}(x)\right)^{2}}{h_{0}^{\varepsilon}(x)}+2 \frac{m_{0}^{\varepsilon}(x)}{h_{0}^{\varepsilon}(x)} \partial_{x} h_{0}^{\varepsilon}(x)+\frac{\left(\partial_{x} h_{0}^{\varepsilon}(x)\right)^{2}}{h_{0}^{\varepsilon}(x)}\right) d x+\int_{\Omega} \frac{\left(h_{0}^{\varepsilon}(x)\right)^{2}}{2 F r^{2}} d x+\int_{\Omega} \frac{\left(\partial_{x} h_{0}^{\varepsilon}(x)\right)^{2}}{2 W_{e}} d x \\
& -\frac{4 \alpha}{R_{e}} \int_{\Omega}\left(1+h_{0}^{\varepsilon}(x)\right) \log \left(\frac{h_{0}^{\varepsilon}(x)}{1+h_{0}^{\varepsilon}(x)}\right) d x
\end{aligned}
$$

As for the BF-dissipative entropy, we would notice first, comparing (1) and (11), that $D(r)=F(r)$, and hence to obtain the BF entropy we should only look after the function $G_{0}$.

$$
\begin{aligned}
G_{0}(A) & =\int_{+\infty}^{A} \int_{+\infty}^{s} \frac{1}{F(r)} d r d s \\
& =\int_{+\infty}^{A} \int_{+\infty}^{s} \frac{1}{r^{2}+r^{3}} d r d s \\
& =\int_{+\infty}^{A} \int_{+\infty}^{s} \frac{1}{r^{2}}+\frac{1}{1+r}-\frac{1}{r} d r d s \\
& =-(1+A) \log \left(\frac{A}{1+A}\right) .
\end{aligned}
$$

Hence, substituting in the general form of the BF-entropy [2], we get

$$
\int_{\Omega}-(1+h(x, t)) \log \left(\frac{h(x, t)}{1+h(x, t)}\right) d x+\int_{0}^{t} \int_{\Omega} \frac{\left(\partial_{x}^{2} h\right)^{2}}{\alpha W e}+\frac{\left(\partial_{x} h\right)^{2}}{\alpha F r^{2}} d x d t \leq-\int_{\Omega}\left(1+h_{0}(x)\right) \log \left(\frac{h_{0}(x)}{1+h_{0}(x)}\right) d x
$$

Multiplying by $\frac{4 \alpha}{R_{e}}$ yields

$$
\begin{aligned}
& \frac{4}{R_{e}}\left[-\alpha \int_{\Omega}(1+h(x, t)) \log \left(\frac{h(x, t)}{1+h(x, t)}\right) d x+\int_{0}^{t} \int_{\Omega} \frac{\left(\partial_{x}^{2} h\right)^{2}}{W e}+\frac{\left(\partial_{x} h\right)^{2}}{F r^{2}} d x d t\right] \\
& \leq-\frac{4 \alpha}{R_{e}} \int_{\Omega}\left(1+h_{0}(x)\right) \log \left(\frac{h_{0}(x)}{1+h_{0}(x)}\right) d x .
\end{aligned}
$$

Due to the weak convergence of the systems and the lack of higher orders of convergence on most of the terms in the BD-entropy, the convergence between the entropies will be exclusively a convergence of inequalities. Hence, using the weakly lower semi continuity property of the $L^{2}$ norm and the weak convergence of the terms stated 
in section 1.2 , we get the convergence of the energy inequality 12 to the following inequality

$$
\frac{1}{2} \int_{\Omega} \frac{h^{2}}{F r^{2}}+\frac{\left(\partial_{x} h\right)^{2}}{W_{e}} d x+\alpha \int_{0}^{t} \int_{\Omega} \frac{h^{2} u^{2}}{F(h)} d x d t \leq C .
$$

As for the Bd-entropy, we obtain the convergence to the following inequality

$$
\begin{aligned}
& \frac{1}{2} \int_{\Omega} \frac{h^{2}}{F r^{2}}+\frac{\left(\partial_{x} h\right)^{2}}{W_{e}} d x-\frac{4 \alpha}{R_{e}}(1+h(x, t)) \log \left(\frac{h(x, t)}{1+h(x, t)}\right) d x \\
& +\alpha \int_{0}^{t} \int_{\Omega} \frac{h^{2} u^{2}}{F(h)}+\frac{4}{R e} \int_{0}^{t} \int_{\Omega} \frac{\left(\partial_{x}^{2} h\right)^{2}}{\alpha W e}+\frac{\left(\partial_{x} h\right)^{2}}{\alpha F r^{2}} d x d t \leq C .
\end{aligned}
$$

Coupling the last inequality with the energy inequality (35) yields

$$
-\alpha \int_{\Omega}(1+h(x, t)) \log \left(\frac{h(x, t)}{1+h(x, t)}\right) d x+\int_{0}^{t} \int_{\Omega} \frac{\left(\partial_{x}^{2} h\right)^{2}}{W e}+\frac{\left(\partial_{x} h\right)^{2}}{F r^{2}} d x d t \leq C,
$$

which obviously resembles the so called BF-entropy inequality that can be noticed from (33).

\section{The Limit of A VISCOUS COMPRESSIBle SYSTEM WITH A GENERAL DRAG TERM}

Following the footsteps of the previous section, but considering a viscous compressible model with a general drag term given by $\alpha h^{2-n} u$ and general pressure term $p(s)=s^{\beta+1} /\left((\beta+1) \mathrm{Fr}^{2}\right)$ namely

$$
\begin{aligned}
& \partial_{t} h_{\varepsilon}+\partial_{x}\left(h_{\varepsilon} u_{\epsilon}\right)=0, \\
& \varepsilon\left(\partial_{t}\left(h_{\varepsilon} u_{\epsilon}\right)+\partial_{x}\left(h_{\varepsilon} u_{\epsilon}^{2}\right)\right)+\frac{1}{F r^{2}} h_{\varepsilon}^{\beta} \partial_{x}\left(h_{\varepsilon}\right)=\varepsilon\left(\frac{4}{R_{e}} \partial_{x}\left(h_{\varepsilon} \partial_{x} u_{\epsilon}\right)\right)+\frac{1}{W_{e}} h_{\varepsilon} \partial_{x}^{3} h_{\varepsilon}-\alpha \frac{h_{\varepsilon}^{2} u_{\epsilon}}{h_{\varepsilon}^{n}},
\end{aligned}
$$

where $\beta+n \in(1,2)$. Such system would converge in the formal sense to the following fourth order lubrication approximation that has been studied in several papers [2]

$$
\begin{aligned}
& \partial_{t} h+\partial_{x}(h u)=0, \\
& h u=\frac{1}{\alpha W_{e}} h^{n} \partial_{x}^{3} h-\frac{1}{\alpha F r^{2}} h^{\beta+n-1} \partial_{x} h .
\end{aligned}
$$

Hence, compared to (1), we get that $F(h)=h^{n}$ and $D(h)=h^{\beta+n-1}$. We will consider in the sequel that $m=\beta+n$. In 2 for instance, the authors considered the above lubrication model with the same choice of $F$ and $D$. They proved the existence of a global in time nonnegative weak solution starting from nonnegative datum for all $n>0$, and $1<m<2$. In particular, The most critical case is the most significantly physical one when $n=3$ (moving contact line in a thin film). In this case, a distributional solution is proven to exist, where it becomes a strong positive solution in the infinite time limit. The BF entropy corresponding to the latter system is given by

$$
\int_{\Omega} G_{0}(h(x, T)) d x+\int_{0}^{T} \int_{\Omega} \frac{\left(\partial_{x}^{2} h\right)^{2}}{\alpha W e}+\frac{1}{\alpha F r^{2}} h^{m-n-1}\left(\partial_{x} h\right)^{2} d x d t=\int_{\Omega} G_{0}\left(h_{0}(x)\right) d x .
$$

A further look on $G_{0}$ allows us distinguish 3 cases: 
(1) $n \neq 1$ and $n \neq 2$

$$
\begin{aligned}
G_{0}(A) & =\int_{C_{1}}^{A} \int_{C_{2}}^{s} \frac{1}{F(r)} d r d s \\
& =\int_{C_{1}}^{A} \int_{C_{2}}^{s} \frac{1}{r^{n}} d r d s \\
& =\int_{C_{1}}^{A} \frac{1}{1-n} s^{1-n} d s \\
& =\frac{1}{(1-n)(2-n)} A^{2-n} .
\end{aligned}
$$

$\left(C_{i}\right)_{i=1,2}$ are chosen such that: $C_{1}=C_{2}=+\infty$ if $n>2$, and $C_{1}=0, C_{2}=+\infty$ when $1<n<2$, $C_{1}=C_{2}=0$ when $n<1$.

(2) $n=1$

$$
\begin{aligned}
G_{0}(A) & =\int_{e}^{A} \int_{1}^{s} \frac{1}{F(r)} d r d s \\
& =\int_{e}^{A} \int_{1}^{s} \frac{1}{r} d r d s \\
& =\int_{e}^{A} \log (s) d s \\
& =A \log (A)-A .
\end{aligned}
$$

(3) $n=2$

$$
\begin{aligned}
G_{0}(A) & =\int_{1}^{A} \int_{+\infty}^{s} \frac{1}{F(r)} d r d s \\
& =\int_{1}^{A} \int_{+\infty}^{s} \frac{1}{r^{2}} d r d s \\
& =\int_{1}^{A} \frac{-1}{s} d s \\
& =-\log (A) .
\end{aligned}
$$

The third case $n=2$ is studied thoroughly in [5], therefore we wouldn't consider it at the moment. In the rest of this part will consider $\mathrm{n}$ is not equal to 1 nor to 2 .

\subsection{Ansatz between $\mathbf{n}$ and $\mathbf{m}$ on the physical basis}

Consider the system (39) that we call PLM ( corresponding to porous-lubrication model). In [2] the authors studied system PLM for the case where $n>0$ and $1<m<2$. Their paper in fact is an outcome of two previous papers [20] and [3; one studying the porous medium problem describing the flow of a gas in a porous medium given by system (41), and the second is the study of the lubrication approximation model of surface tension dominated motion given by system 42 :

$$
\begin{gathered}
\partial_{t} h+\partial_{x}^{2}\left(h^{m}\right)=0, \\
\partial_{t} h+\partial_{x}\left(h^{n} \partial_{x}^{3} h\right)=0 .
\end{gathered}
$$

Physically such systems model droplets spreading on a solid interface. Consider just a wetting case, the liquid is characterized by a positive spreading constant and thus the drops tend to wet the surface totally. In this case, intermolecular forces near the interface are important to account for in the dynamics of the system. Technically, this can be represented by a cut-off of the disjoint pressure at the molecular length scale which is interpreted as a second order term, or Van Der Waals term or porous medium term in the mass conservation evolution 
equation. This in fact may explain the convergence at the physical basis of the Shallow water model into the lubrication model as for instance the case of thin films at the nanoscale or droplets array spreading. In such cases, the effect of capillarity dominating the motion, and the spreading manner which resembles the manner that a liquid spreads in a porous medium explain that the motion would emanate from a drag and pressure forces in (38) as well as a capillarity effect contribution.

It is good to remark that in (41), the existence of solution as well as regularity is proven for a more general choice of $\mathrm{m}$, but in accordance with LM in (42), only for $1<m<2$ that PM possess a source type solution that touches down with a zero slope which coincides with a similar manner to the solution of LM. An important discussion here is the competition as a function of $\mathrm{n}$ and $\mathrm{m}$ between the porous medium term which is represented by a second order derivative term in the evolution equation, and the capillarity effect represented by the fourth order term. For $n>0$ and $1<m<2$, an asymptotic analysis via leading order of transport waves at the edge of the support of the solution is applied and predicts a kind of power law behavior of the solution at the edge. The results are clearly manifested in [2], and the reader can refer to table 1 in the paper for a brief summary of the competition with respect to the relation between $\mathrm{n}$ and $\mathrm{m}$.

\subsection{Mathematical justification}

Consider the energy and BD entropy inequalities of system 38

$$
\begin{aligned}
& \frac{1}{2}\left(\int_{\Omega} \varepsilon h_{\varepsilon}(x, t) u_{\epsilon}^{2}(x, t)+\frac{1}{F r^{2}} \frac{h_{\varepsilon}(x, t)^{\beta+1}}{\beta(\beta+1)}+\frac{\left(\partial_{x} h_{\varepsilon}(x, t)\right)^{2}}{W_{e}}\right) d x+\int_{0}^{t} \int_{\Omega} \frac{4 \varepsilon}{R_{e}} h_{\varepsilon}\left(\partial_{x} u_{\epsilon}\right)^{2}+\alpha \frac{h_{\varepsilon}^{2} u_{\epsilon}^{2}}{h_{\varepsilon}^{n}} d x d t \\
& \leq \frac{1}{2} \int_{\Omega} \varepsilon \frac{\left(m_{0}^{\varepsilon}\right)^{2}}{h_{0}^{\varepsilon}}+\frac{1}{F r^{2}} \frac{\left(h_{0}^{\varepsilon}\right)^{\beta+1}}{\beta(\beta+1)}+\frac{\left(\partial_{x} h_{0}^{\varepsilon}\right)^{2}}{W_{e}} d x .
\end{aligned}
$$

and

$$
\begin{aligned}
& \frac{1}{2} \int_{\Omega} \varepsilon h_{\varepsilon}(x, t) v_{\varepsilon}(x, t)^{2}+\frac{1}{F_{r}^{2}} \frac{h_{\varepsilon}(x, t)^{\beta+1}}{\beta(\beta+1)}+\frac{\left(\partial_{x} h_{\varepsilon}(x, t)\right)^{2}}{W_{e}} d x+\frac{4 \alpha}{R e} \int_{\Omega} \frac{1}{(1-n)(2-n)} h_{\varepsilon}(x, t)^{2-n} d x \\
& +\frac{4}{R e}\left[\int_{0}^{t} \int_{\Omega} \frac{1}{F r^{2}} h_{\varepsilon}^{\beta-1}\left(\partial_{x} h_{\varepsilon}\right)^{2} d x+\frac{1}{W_{e}}\left(\partial_{x}^{2} h_{\varepsilon}\right)^{2} d x d t\right]+\alpha \int_{0}^{t} \int_{\Omega} \frac{h_{\varepsilon}^{2} u_{\epsilon}^{2}}{h_{\varepsilon}^{n}} d x d t \\
& \leq \frac{1}{2} \int_{\Omega}\left(\varepsilon \frac{\left(m_{0}^{\varepsilon}\right)^{2}}{h_{0}^{\varepsilon}}+2 \frac{m_{0}^{\varepsilon}}{h_{0}^{\varepsilon}} \partial_{x} h_{0}^{\varepsilon}(x)+\frac{\left(\partial_{x} h_{0}^{\varepsilon}(x)\right)^{2}}{h_{0}^{\varepsilon}(x)}\right) d x+\frac{1}{2} \int_{\Omega}\left(\frac{1}{F r^{2}} \frac{\left(h_{0}^{\varepsilon}\right)^{\beta+1}}{\beta(\beta+1)}+\frac{\left(\partial_{x} h_{0}^{\varepsilon}\right)^{2}}{W_{e}}\right) d x \\
& +\frac{4 \alpha}{R e} \int_{\Omega} \frac{1}{(1-n)(2-n)}\left(h_{0}^{\varepsilon}\right)^{2-n}(x) d x
\end{aligned}
$$

Definition 2.1. A weak solution of system (38) is a couple $(h, u)$ satsifying the following integral equations as well as the energy and BD-entropy inequality given in (43) and (44)

$$
\int_{0}^{\infty} \int_{\Omega} h_{\varepsilon} \partial_{t} \psi d x d t+\int_{\Omega} h_{0}^{\varepsilon} \psi(\cdot, 0) d x=-\int_{0}^{\infty} \int_{\Omega} h_{\varepsilon} u_{\epsilon} \partial_{x} \psi d x d t
$$

and

$$
\begin{aligned}
& \varepsilon\left(\int_{0}^{\infty} \int_{\Omega} h_{\varepsilon} u_{\epsilon} \partial_{t} \phi d x d t+\int_{\Omega} m_{0}^{\varepsilon} \phi(\cdot, 0) d x+\int_{0}^{\infty} \int_{\Omega} h_{\varepsilon} u_{\epsilon}^{2} \partial_{x} \phi d x d t\right) \\
& -\frac{4 \varepsilon}{R_{e}} \int_{0}^{\infty} \int_{\Omega} h_{\varepsilon} \partial_{x} u_{\epsilon} \partial_{x} \phi d x d t+\frac{1}{W_{e}} \int_{0}^{\infty} \int_{\Omega} \frac{1}{2}\left(\partial_{x} h_{\varepsilon}\right)^{2} \partial_{x} \phi d x d t \\
& -\frac{1}{W_{e}} \int_{0}^{\infty} \int_{\Omega} h_{\varepsilon} \partial_{x}^{2} h_{\varepsilon} \partial_{x} \phi d x d t+\frac{1}{F r^{2}} \int_{0}^{\infty} \int_{\Omega} \frac{1}{\beta+1} h_{\varepsilon}^{\beta+1} \partial_{x} \phi d x d t-\alpha \int_{0}^{\infty} \int_{\Omega} \frac{h_{\varepsilon}^{2} u_{\epsilon}}{h_{\varepsilon}^{n}} \phi d x d t=0
\end{aligned}
$$


for all $\psi \in \mathcal{C}_{0}^{\infty}(\Omega \times[0, \infty))$ and $\phi \in \mathcal{C}_{0}^{\infty}(\Omega \times[0, \infty))$.

We can prove the global existence of weak solutions following the recent papers by [21, 19], 11]. For more details, readers may consult [9] where the authors explain the procedures in details. Remark that the new quantities here concern drag terms which are compatible with the analysis.

Theorem 2.1. Let $\left(h_{0}^{\varepsilon}, m_{0}^{\varepsilon}\right)$ be such that $h_{0}^{\varepsilon} \geq 0$ and

$$
\begin{gathered}
\varepsilon \frac{\left(m_{0}^{\varepsilon}\right)^{2}}{h_{0}^{\varepsilon}} \in L^{1}(\Omega), \quad h_{0}^{\varepsilon} \in H^{1}(\Omega), \\
\sqrt{\varepsilon} \partial_{x}\left(\sqrt{h_{0}^{\varepsilon}}\right) \in L^{2}(\Omega), \quad\left(h_{0}^{\varepsilon}\right)^{2-n} \in L^{1}(\Omega) .
\end{gathered}
$$

Then there exists a global weak solution of (38) in the sense of Definition 2.1.

Theorem 2.2. Suppose there exits a sequence $\left(h_{\varepsilon}, u_{\epsilon}\right)$ a weak solution of system (38) in the sense of definition 2.1 given by Theorem 2.1. then, up to a subsequence, this couple converges in a weak sense to the weak solution $(h, u)$ of the lubrication model given in system (39).

Proof. We will sketch the proof briefly since it follows that of theorem 1.2

From (43) and 44), we can deduce the following uniform bounds accounting for initial data satisfying the bounds in theorem 2.1

$$
\begin{aligned}
& \sqrt{\varepsilon}\left\|\sqrt{h}_{\varepsilon} u_{\epsilon}\right\|_{L^{\infty}\left(0, T ; L^{2}(\Omega)\right)} \leq C, \quad\left\|h_{\varepsilon}^{\frac{\beta+1}{2}}\right\|_{L^{\infty}\left(0, T ; L^{2}(\Omega)\right)} \leq C, \quad\left\|\partial_{x} h_{\varepsilon}\right\|_{L^{\infty}\left(0, T ; L^{2}(\Omega)\right)} \leq C, \\
& \sqrt{\varepsilon}\left\|\sqrt{h}_{\varepsilon} \partial_{x} u_{\epsilon}\right\|_{L^{2}\left(0, T ; L^{2}(\Omega)\right)} \leq C, \quad\left\|h_{\varepsilon}^{\frac{2-n}{2}} u_{\epsilon}\right\|_{L^{2}\left(0, T ; L^{2}(\Omega)\right)} \leq C, \quad \sqrt{\varepsilon}\left\|\sqrt{h_{\varepsilon}} v_{\varepsilon}\right\|_{L^{\infty}\left(0, T ; L^{2}(\Omega)\right)} \leq C, \\
& \left\|h_{\varepsilon}^{\frac{2-n}{2}}\right\|_{L^{\infty}\left(0, T ; L^{2}(\Omega)\right)} \leq C, \quad\left\|\partial_{x}\left(h_{\varepsilon}^{\frac{\beta+1}{2}}\right)\right\|_{L^{2}\left(0, T ; L^{2}(\Omega)\right)} \leq C, \quad\left\|\partial_{x}^{2} h_{\varepsilon}\right\|_{L^{2}\left(0, T ; L^{2}(\Omega)\right)} \leq C
\end{aligned}
$$

Regarding that $h_{\varepsilon}$ is in $L^{\infty}\left(0, T ; L^{1}(\Omega)\right)$ from the conservation of mas (mass equation), we can infer the following using Poincare's inequality

$$
\begin{aligned}
\left\|h_{\varepsilon}\right\|_{L^{2}(\Omega)} & \leq\left\|h_{\varepsilon}-\overline{h_{\varepsilon}}\right\|_{L^{2}(\Omega)}+\left\|\overline{h_{\varepsilon}}\right\|_{L^{2}(\Omega)} \\
& \leq\left\|\partial_{x} h_{\varepsilon}\right\|_{L^{2}(\Omega)}+C\left\|h_{\varepsilon}\right\|_{L^{1}(\Omega)} \\
& \leq C,
\end{aligned}
$$

where $\overline{h_{\varepsilon}}=\frac{1}{|\Omega|} \int_{\Omega} h_{\varepsilon} d x$. Hence

$$
h_{\varepsilon} \in L^{\infty}\left(0, T ; H^{1}(\Omega)\right) .
$$

Due to compactness we get as well

$$
h_{\varepsilon} \in L^{\infty}((0, T) \times \Omega) .
$$

Again, by compactness results we find that $h_{\varepsilon}$ converges strongly to some $h$ in $C\left([0, T], L^{2}(\Omega)\right)$. Thus we get

$$
h_{\varepsilon}(x, 0) \rightarrow h(x, 0) \quad \text { in } \quad L^{2}(\Omega) .
$$

We denote by $h_{0}$ the weak limit in $L^{2}(\Omega)$ of $h_{0}^{\varepsilon}$. Due to uniform boundedness of $\partial_{x}^{2} h_{\varepsilon}$ in $L^{2}\left(0, T ; L^{2}(\Omega)\right)$ we get that $h_{\varepsilon}$ is bounded uniformly in $L^{2}\left(0, T ; H^{2}(\Omega)\right)$, and using Aubin's compactness theorem, we deduce that $h_{\varepsilon}$ would converge strongly to $h$ in $L^{2}\left(0, T ; H^{1}(\Omega)\right)$.

Now, we are concerned with the convergence of the velocity field in $(38)$. In fact, we can argue here for two situations: the first is when $n>2$, and the second is when $0<n<2$. In the first case, the proof follows exactly the steps in the proof of theorem 2.1 so that we get $u_{\epsilon}$ converges weakly to some function $u$ in $L^{2}\left(0, T ; L^{2}(\Omega)\right)$. For $0<n<2$, we will prove first the convergence of the momentum following a similar approach to that introduced by the authors in 7 using the presence of the drag term mainly using the uniform bounds from 
energy and BD-entropy inequalities.

The term $h_{\varepsilon} u_{\epsilon}$ can be rewritten as

$$
h_{\varepsilon} u_{\epsilon}=h_{\varepsilon}^{\frac{n}{2}} h_{\varepsilon}^{\frac{2-n}{2}} u_{\epsilon}
$$

From the energy estimates, we have $h_{\varepsilon}^{\frac{2-n}{2}} u_{\epsilon}$ is uniformly bounded in $L^{2}((0, T) \times \Omega)$ and thus it converges weakly in such space to a function that we will denote $\xi$. Now since $n>0$, then $h_{\varepsilon}^{\frac{n}{2}}$ is bounded in $L^{\infty}((0, T) \times \Omega)$, which implies that the momentum is bounded uniformly in $L^{2}((0, T) \times \Omega)$. Thus this insures also that $h_{\varepsilon} u_{\epsilon}$ converges weakly to some function in $L^{2}((0, T) \times \Omega)$. Besides, for $\varepsilon$ large enough, we may assume that on the set $\{(x, t) \in(0, \infty) \times \Omega / h(x, t)=0\} h_{\varepsilon}$ is taken equal to zero, and thus $\xi$ is also equals to zero on such set. Now we can define the function $u$ such that

$$
u=\left\{\begin{array}{lr}
\frac{\xi}{h^{\frac{2-n}{2}}} & h \neq 0, \\
0 & \text { elsewhere }
\end{array}\right.
$$

We claim now that the momentum $h_{\varepsilon} u_{\epsilon}$ converges weakly in $L^{2}((0, T) \times \Omega)$ to $h u$. In fact, we have

$$
\int_{0}^{t} \int_{\Omega}\left(h_{\varepsilon} u_{\epsilon}-h u\right) \psi d x d t=\int_{0}^{t} \int_{\Omega} h^{\frac{n}{2}}\left(h_{\varepsilon}^{\frac{2-n}{2}} u_{\epsilon}-h^{\frac{2-n}{2}} u\right) \psi d x d t+\int_{0}^{t} \int_{\Omega} h_{\varepsilon}^{\frac{2-n}{2}} u_{\epsilon}\left(h_{\varepsilon}^{\frac{n}{2}}-h^{\frac{n}{2}}\right) \psi d x d t
$$

As for the first term on the right hand side, it converges to zero due to weak convergence of the drag term in $L^{2}((0, T) \times \Omega)$. The second term converges to zero as well using almost everywhere convergence of $h_{\varepsilon}$ to $h$ and a LDCT approach.

Remark here that $u$ is not defined uniquely on the set $\{(x, t) \in(0, \infty) \times \Omega / h(x, t)=0\}$.

Once obtained enough convergence results, we are able now to pass to the limit in the weak formulations 45 and (46) following the same analysis as in the proof of theorem 1.2. As for the weak formulation of the mass equation, we use the strong convergence of $h_{\varepsilon}$ to $h$ in $L^{2}\left(0, T ; L^{2}(\Omega)\right)$ as well as the weak convergence of $u_{\epsilon}$ to $u$ in $L^{2}\left(0, T ; L^{2}(\Omega)\right)$ in case $n>2$, or the weak convergence of the momentum in $L^{2}((0, T) \times \Omega)$ in case $0<n<2$. Concerning the terms multiplied by $\varepsilon$ in the second integral equation of the weak formulation, the uniform boundedness in $\varepsilon$ of such terms allows them to converge to zero as $\varepsilon$ tends to zero (using the estimates obtained at the beginning of the proof). As for the fifth and sixth terms which come from the capillarity term in system (38), the convergence comes as a result of the strong convergence of $h_{\varepsilon}$ to $h$ in $L^{2}\left(0, T ; H^{1}(\Omega)\right)$ and the weak convergence $\partial_{x}^{2} h_{\varepsilon}$ to $\partial_{x}^{2} h$ in $L^{2}\left(0, T ; L^{2}(\Omega)\right)$. Using LDCT and the fact that the strong convergence of $h_{\varepsilon}$ implies almost everywhere convergence up to a subsequence, we can prove the convergence of the seventh term as well. Finally, for the last term corresponding to the drag term, it follows from its weak convergence to $\xi=h^{\frac{2-n}{2}} u$ in $L^{2}((0, T) \times \Omega)$ for $0<n<2$ (otherwise its convergence follows as in the proof of theorem 1.2 . Therefore, we get that the weak limit couple $(h, u)$ is in fact a weak solution of the lubrication system since it satisfies its corresponding weak formulation:

$$
\begin{aligned}
& \int_{0}^{\infty} \int_{\Omega} h \partial_{t} \psi d x d t+\int_{\Omega} h_{0} \psi(\cdot, 0) d x=-\int_{0}^{\infty} \int_{\Omega} h u \partial_{x} \psi d x d t \\
& \frac{1}{W_{e}} \int_{0}^{\infty} \int_{\Omega} \frac{1}{2}\left(\partial_{x} h\right)^{2} \partial_{x} \phi d x d t-\frac{1}{W_{e}} \int_{0}^{\infty} \int_{\Omega} h \partial_{x}^{2} h_{\varepsilon} \partial_{x} \phi d x d t \\
& +\frac{1}{F r^{2}} \int_{0}^{\infty} \int_{\Omega} \frac{1}{\beta+1} h^{\beta+1} \partial_{x} \phi d x d t-\alpha \int_{0}^{\infty} \int_{\Omega} \frac{h^{2} u}{h^{n}} \phi d x d t=0 .
\end{aligned}
$$




\subsection{Convergence of the BD-entropy}

Using the uniform bounds of the initial datum, we recall that the energy and BD-entropy inequalities of system (9) is given by

$$
\begin{aligned}
& \frac{1}{2}\left(\int_{\Omega} \varepsilon h_{\varepsilon}(x, t) u_{\epsilon}^{2}(x, t)+\frac{1}{F r^{2}} \frac{h_{\varepsilon}(x, t)^{\beta+1}}{\beta(\beta+1)}+\frac{\left(\partial_{x} h_{\varepsilon}(x, t)\right)^{2}}{W_{e}}\right) d x+\int_{0}^{t} \int_{\Omega} \frac{4 \varepsilon}{R_{e}} h_{\varepsilon}\left(\partial_{x} u_{\epsilon}\right)^{2}+\alpha \frac{h_{\varepsilon}^{2} u_{\epsilon}^{2}}{h_{\varepsilon}^{n}} d x d t \leq C . \\
& \quad \frac{1}{2} \int_{\Omega} \varepsilon h_{\varepsilon}(x, t) v_{\varepsilon}(x, t)^{2}+\frac{1}{F_{r}^{2}} \frac{h_{\varepsilon}(x, t)^{\beta+1}}{\beta(\beta+1)}+\frac{\left(\partial_{x} h_{\varepsilon}(x, t)\right)^{2}}{W_{e}} d x+\frac{4 \alpha}{R e} \int_{\Omega} \frac{1}{(1-n)(2-n)} h_{\varepsilon}(x, t)^{2-n} d x \\
& \quad+\frac{4}{R e}\left[\int_{0}^{t} \int_{\Omega} \frac{1}{F r^{2}} h_{\varepsilon}^{\beta-1}\left(\partial_{x} h_{\varepsilon}\right)^{2} d x+\frac{1}{W_{e}}\left(\partial_{x}^{2} h_{\varepsilon}\right)^{2} d x d t\right]+\alpha \int_{0}^{t} \int_{\Omega} \frac{h_{\varepsilon}^{2} u_{\epsilon}^{2}}{h_{\varepsilon}^{n}} d x d t \leq C .
\end{aligned}
$$

Using the weakly lower semi continuity property of the $L^{2}$ norm and the weak convergence of the different terms in the above two inequalities, we can deduce the following

$$
\begin{gathered}
\frac{1}{2}\left(\int_{\Omega} \frac{1}{F r^{2}} \frac{h(x, t)^{\beta+1}}{\beta(\beta+1)}+\frac{\left(\partial_{x} h(x, t)\right)^{2}}{W_{e}}\right) d x+\alpha \int_{0}^{t} \int_{\Omega} \frac{h^{2} u^{2}}{h^{n}} d x d t \leq C . \\
\frac{1}{2} \int_{\Omega} \frac{1}{F_{r}^{2}} \frac{h(x, t)^{\beta+1}}{\beta(\beta+1)}+\frac{\left(\partial_{x} h(x, t)\right)^{2}}{W_{e}} d x+\frac{4 \alpha}{R e} \int_{\Omega} \frac{1}{(1-n)(2-n)} h(x, t)^{2-n} d x \\
+\frac{4}{R e}\left[\int_{0}^{t} \int_{\Omega} \frac{1}{F r^{2}} h^{\beta-1}\left(\partial_{x} h\right)^{2} d x+\frac{1}{W_{e}}\left(\partial_{x}^{2} h\right)^{2} d x d t\right]+\alpha \int_{0}^{t} \int_{\Omega} \frac{h^{2} u^{2}}{h^{n}} d x d t \leq C .
\end{gathered}
$$

Coupling the above two inequalities together yields

$$
\frac{4 \alpha}{R e} \int_{\Omega} \frac{1}{(1-n)(2-n)} h(x, t)^{2-n} d x+\frac{4}{R e} \int_{0}^{t} \int_{\Omega} \frac{1}{F r^{2}} h^{\beta-1}\left(\partial_{x} h\right)^{2} d x+\frac{1}{W_{e}}\left(\partial_{x}^{2} h\right)^{2} d x d t \leq C,
$$

which coincides with the BF-dissipative entropy of system (39) given by inequality 40 . Once again we can say that the BD entropy of shallow water system converges in a weak sense to the BF-dissipative entropy of the corresponding lubrication system.

\section{A MORE GENERAL FRAMEWORK.}

Consider the following logarithmic fourth-order equation:

$$
\partial_{t} h+\partial_{x}^{2}\left(F(h) \partial_{x}^{2} G(h)\right)=0
$$

On the other hand we consider the following viscous compressible model with capillarity and drag term (neglecting pressure) given by

$$
\begin{aligned}
\partial_{t} h_{\varepsilon}+\partial_{x}\left(h_{\varepsilon} u_{\epsilon}\right)=0 \\
\begin{aligned}
\varepsilon\left(\partial_{t}\left(h_{\varepsilon} u_{\epsilon}\right)+\partial_{x}\left(h_{\varepsilon} u_{\epsilon}^{2}\right)+\partial_{x} p\left(h_{\varepsilon}\right)\right)=\varepsilon & \left(\frac{4}{\mathrm{R}_{\mathrm{e}}} \partial_{x}\left(\lambda\left(h_{\varepsilon}\right) \partial_{x} u_{\epsilon}\right)\right) \\
& +\frac{1}{\mathrm{~W}_{\mathrm{e}}} h_{\varepsilon} \partial_{x}\left(\sqrt{K\left(h_{\varepsilon}\right)} \partial_{x}^{2}\left(\int_{0}^{h_{\varepsilon}} \sqrt{K(r)} d r\right)\right)-h_{\varepsilon} u_{\epsilon} .
\end{aligned}
\end{aligned}
$$

where $p(s)=a s^{\gamma}$ with $a>0$ and $\gamma>1$ two fixed parameter, Re and We respectively the Reynolds and Weissenberg coefficients and with initial data

$$
\left.h_{\varepsilon}\right|_{t=0}=h_{0}^{\varepsilon},\left.\quad h_{\varepsilon} u_{\epsilon}\right|_{t=0}=m_{0}^{\varepsilon} .
$$


The viscosity function $\lambda$ is supposed to embed a shear-type viscosity $\mu(h)=\int_{0}^{h} \frac{\lambda(r)}{2 r} d r$. In fact, a link indispensably relating between the viscosity $\mu$ and the capillarity $K$ (to be able to follow existence result recently obtained in [11]) is given by

$$
\mu^{\prime}(h)=\frac{1}{2} \sqrt{h} \sqrt{K(h)}
$$

Define now

$$
S(h)=\int_{0}^{h} \frac{\sqrt{K(r)}}{\sqrt{r}} d r, \quad Z(h)=\int_{0}^{h} \frac{\mu^{\prime}(r) \sqrt{\mu(r)}}{r} d r .
$$

Furthemore, we will assume in the sequel that $\mu$ satisfies the following hypothesis:

$$
\mu \in \mathcal{C}^{0}\left(\mathbb{R}_{+} ; \mathbb{R}_{+}\right) \cap \mathcal{C}^{2}\left(\mathbb{R}_{+}^{*} ; \mathbb{R}\right)
$$

There exists $a_{1}, a_{2}$ and some constant $C$ such that

$$
\begin{gathered}
0<\frac{2}{3}<a_{1}<a_{2}<4 \\
0<\frac{h \mu^{\prime}(h)}{a_{2}} \leq \mu(h) \leq \frac{h \mu^{\prime}(h)}{a_{1}} \quad \forall h>0,
\end{gathered}
$$

and

$$
\left|\frac{h \mu^{\prime \prime}(h)}{\mu^{\prime}(h)}\right| \leq C<+\infty .
$$

At the formal level, system (55) converges to the following system

$$
\begin{aligned}
& \partial_{t} h+\partial_{x}(h u)=0, \\
& h u=\frac{1}{\mathrm{~W}_{\mathrm{e}}} h \partial_{x}\left(\sqrt{K(h)} \partial_{x}^{2}\left(\int_{0}^{h} \sqrt{K(r)} d r\right)\right),
\end{aligned}
$$

which can be written as

$$
\partial_{t} h+\partial_{x}\left(\frac{1}{\mathrm{~W}_{\mathrm{e}}} h \partial_{x}\left(\sqrt{K(h)} \partial_{x}^{2}\left(\int_{0}^{h} \sqrt{K(r)} d r\right)\right)\right)=0 .
$$

Using Bohm's identity proven in 6 we have

$$
h \partial_{x}\left(\sqrt{K(h)} \partial_{x}^{2}\left(\int_{0}^{h} \sqrt{K(r)} d r\right)\right)=\partial_{x}\left(h \gamma^{\prime}(h) \partial_{x}^{2} \theta(h)\right)
$$

where $\gamma^{\prime}(h)=\sqrt{h K(h)}$ and $\theta^{\prime}(h)=2 \sqrt{\frac{K(h)}{h}}$. In accordance with our definition in 566), we get that $\gamma(h)=$ $2 \mu(h)$, and $\theta(h)=2 S(h)$. Thus substituting the identity in equation 62 we obtain the following

$$
\partial_{t} h+\partial_{x}^{2}\left(2 h \mu^{\prime}(h) \partial_{x}^{2}(2 S)\right)=0
$$

Which recovers equation (54) taking $F(h)=2 h \mu^{\prime}(h)$ and $G(h)=2 S$. In the sequel, we will define $\Psi^{\prime}(h)=$ $\sqrt{K(h)}$ for simplicity. The global existence of weak solutions for the compressible system with capillarity and drag terms follows quite similar lines than for the approximate system in [11. We will not rewrite the proof because it is not really the objective of the paper which concerns the link between Equation 54 and System 55 with their corresponding initial data. 


\subsection{Limit problem}

Following the same framework based on the energy estimations as in the previous section, we will start from a global weak solution (whence nonnegative) of the shallow water model (55). The energy and BD-entropy of the corresponding system are given respectively by

$$
\begin{aligned}
& \sup _{t \in(0, T)}\left[\frac{1}{2}\left(\int_{\Omega} \varepsilon h_{\varepsilon} u_{\epsilon}^{2}+\left(\partial_{x} \Psi(h)\right)^{2} d x\right)(t)+\int_{\Omega} \varepsilon \Pi\left(h_{\varepsilon}\right) d x\right]+\int_{0}^{T} \int_{\Omega} h_{\varepsilon} u_{\epsilon}^{2}+\varepsilon 2 h_{\varepsilon} \mu^{\prime}\left(h_{\varepsilon}\right)\left(\partial_{x} u_{\epsilon}\right)^{2} d x \\
& \leq \frac{1}{2} \int_{\Omega} \varepsilon \frac{m_{0}^{\varepsilon}}{h_{0}^{\varepsilon}}+\left(\partial_{x} \Psi\left(h_{0}^{\varepsilon}\right)\right)^{2} d x+\int_{\Omega} \varepsilon \Pi\left(h_{0}^{\varepsilon}\right) d x \\
& \sup _{t \in(0, T)}\left[\frac{1}{2} \frac{d}{d t} \int_{\Omega} \varepsilon h_{\varepsilon} v_{\varepsilon}^{2}+M\left(h_{\varepsilon}\right)+\left(\partial_{x} \Psi(h)\right)^{2} d x+\int_{\Omega} \varepsilon \Pi\left(h_{\varepsilon}\right) d x\right]+\int_{\Omega} h_{\varepsilon} u_{\epsilon}^{2}+h_{\varepsilon} \mu^{\prime}\left(h_{\varepsilon}\right)\left(\partial_{x}^{2}(2 S)\right)^{2} d x \\
& +2 \varepsilon \int_{0}^{T} \int_{\Omega} p^{\prime}\left(h_{\varepsilon}\right) S^{\prime}\left(h_{\varepsilon}\right)\left|\partial_{x} h_{\varepsilon}\right|^{2} \leq \frac{1}{2} \int_{\Omega} \varepsilon h_{0}^{\varepsilon}\left|v_{0}^{\varepsilon}\right|^{2}+\left(\partial_{x} \Psi\left(h_{0}^{\varepsilon}\right)\right)^{2} d x+\int_{\Omega} \varepsilon \Pi\left(h_{0}^{\varepsilon}\right) d x,
\end{aligned}
$$

with $\Pi(s)=s \int_{a}^{s} p(\tau) / \tau^{2} d \tau$ where $v_{\varepsilon}=u_{\epsilon}+\partial_{x}(2 S)$. The new term $m\left(h_{\varepsilon}\right)$ is a drag descent term, as we multiply by $\partial_{x}(2 s)$ for the sake of BD-entropy, we come across the following term $\int_{\Omega} h_{\varepsilon} u_{\epsilon} \partial_{x}(2 s) d x$ which can be written as follows:

$$
\begin{aligned}
\int_{\Omega} h_{\varepsilon} u_{\epsilon} \partial_{x}(2 s) d x & =\int_{\Omega} h_{\varepsilon} u_{\epsilon} \partial_{x}\left(4 \int_{1}^{r} \frac{\mu^{\prime}}{r} d r\right) d x \\
& =\int_{\Omega} \partial_{t} h_{\varepsilon}\left(4 \int_{1}^{r} \frac{\mu^{\prime}}{r} d r\right) d x
\end{aligned}
$$

Thus we define $M(h)=\int_{1}^{h} \int_{1}^{r} 4 \frac{\mu^{\prime}}{s} d s d r$. But due to monotonicity of $\mu$ over $(0, \infty)\left(\mu^{\prime}>0\right)$, we can assume that $\mu^{\prime}(\zeta)>\lim _{h \rightarrow 0} \mu^{\prime}(h)=\stackrel{C}{ }$ and $\stackrel{S}{C}<\infty$. Hence we have that:

$$
M(h) \geq C \int_{1}^{h} \int_{1}^{r} \frac{1}{s} d s d r=C(h \log h-h) .
$$

Hence we recover the following entropy:

$$
\begin{aligned}
& \frac{1}{2} \int_{\Omega} \varepsilon h_{\varepsilon} v_{\varepsilon}^{2}+\left(h_{\varepsilon} \log h_{\varepsilon}-h_{\varepsilon}\right)_{+}+\left(\partial_{x} \Psi\right)^{2} d x+\int_{0}^{T} \int_{\Omega} h_{\varepsilon} u_{\epsilon}^{2}+h_{\varepsilon} \mu^{\prime}\left(h_{\varepsilon}\right)\left(\partial_{x}^{2}(2 S)\right)^{2} d x d t \\
& =\frac{1}{2} \int_{\Omega} \varepsilon h_{\varepsilon}^{0}\left(v_{\varepsilon}^{0}\right)^{2}+\left(h_{\varepsilon}^{0} \log h_{\varepsilon}^{0}-h_{\varepsilon}^{0}\right)_{+}+\left(\partial_{x} \Psi_{0}\right)^{2} d x-\int_{\Omega}\left(h_{\varepsilon} \log h_{\varepsilon}-h_{\varepsilon}\right)_{-} d x \leq C d x
\end{aligned}
$$

where we used the fact that $-\int_{\Omega}\left(h_{\varepsilon} \log h_{\varepsilon}-h_{\varepsilon}\right)_{-}$is a positive quantity that does not vanish whenever $h_{\varepsilon}$ belongs to the set $\{x<e\}$ and is bounded by 1 . Thus we can write

$$
-\int_{\Omega} h_{\varepsilon}\left(\log h_{\varepsilon}-1\right)_{-} d x<C
$$

Furthermore, using the logarithmic Sobolev inequality proved in [11, we can obtain some additional estimates from the bounded term $h_{\varepsilon} \mu^{\prime}\left(h_{\varepsilon}\right)\left(\partial_{x}^{2}(2 S)\right)^{2}$ in $L^{2}(0, T ; \Omega)$. We recall the following lemma:

Lemma 3.1. We suppose that $\mu$ satisfies the assumptions (57)-60), then for all $h>0$, and $h \in L^{2}\left(0, T ; H^{2}(\Omega)\right)$, there exists some constant $\alpha$ such that we have the following estimate

$$
\int_{0}^{T} \int_{\Omega}\left(\partial_{x}^{2} Z(h)\right)^{2} d x d t+\alpha \int_{0}^{T} \int_{\Omega} \frac{h^{2}}{\mu(h)^{3}}\left(\partial_{x} h\right)^{4} d x d t \leq \frac{C}{\alpha} \int_{0}^{T} \int_{\Omega} \mu(h)\left(\partial_{x}^{2} S(h)\right)^{2} d x
$$


Finally, we can obtain the following entropy

$$
\int_{\Omega} \frac{\varepsilon}{2} h_{\varepsilon} v_{\varepsilon}^{2}+h_{\varepsilon}\left(\log h_{\varepsilon}-1\right)_{+}+\left(\partial_{x} \Psi\right)^{2} d x+\int_{0}^{T} \int_{\Omega} h_{\varepsilon} u_{\epsilon}^{2}+\left(\partial_{x}^{2} Z\left(h_{\varepsilon}\right)\right)^{2} d x d t \leq C
$$

The estimations obtained from 64 and $(68)$ :

$$
\begin{gathered}
\sqrt{\varepsilon}\left\|\sqrt{h_{\varepsilon}} u_{\epsilon}\right\|_{L^{\infty}\left(0, T ; L^{2}(\Omega)\right)} \leq C, \quad\left\|\partial_{x} \Psi\right\|_{L^{\infty}\left(0, T ; L^{2}(\Omega)\right)} \leq C, \\
\sqrt{\varepsilon}\left\|\sqrt{h_{\varepsilon} \mu^{\prime}} \partial_{x} u_{\epsilon}\right\|_{L^{2}\left(0, T ; L^{2}(\Omega)\right)} \leq C, \quad\left\|\sqrt{h_{\varepsilon}} u_{\epsilon}\right\|_{L^{2}\left(0, T ; L^{2}(\Omega)\right)} \leq C, \\
\left\|\partial_{x} Z\left(h_{\varepsilon}\right)\right\|_{L^{2}\left(0, T ; H^{1}(\Omega)\right)} \leq C, \quad \sqrt{\varepsilon}\left\|\sqrt{h_{\varepsilon}} v_{\varepsilon}\right\|_{L^{\infty}\left(0, T ; L^{2}(\Omega)\right)} \leq C, \\
\left\|h_{\varepsilon}\left(\log h_{\varepsilon}-1\right)_{+}\right\|_{L^{\infty}\left(0, T ; L^{1}(\Omega)\right)} \leq C .
\end{gathered}
$$

1-Uniform boundedness of $\mu\left(h_{\varepsilon}\right)$. Using mass conservation equation, we can write a transport equation on $\mu\left(h_{\varepsilon}\right)$ :

$$
\partial_{t}\left(\mu\left(h_{\varepsilon}\right)\right)+\partial_{x}\left(h_{\varepsilon} \mu^{\prime}\left(h_{\varepsilon}\right) u_{\epsilon}\right)-h_{\varepsilon} \mu^{\prime \prime}\left(h_{\varepsilon}\right) u_{\epsilon} \partial_{x} h_{\varepsilon}=0
$$

Integrating in space and using the fact that $h_{\varepsilon} \mu^{\prime \prime}\left(h_{\varepsilon}\right) \leq C \mu^{\prime}\left(h_{\varepsilon}\right)$ we get

$$
\frac{d}{d t} \int_{\Omega} \mu\left(h_{\varepsilon}\right) d x \leq \int_{\Omega}\left|\frac{\partial_{x} \mu}{\sqrt{h_{\varepsilon}}} \sqrt{h_{\varepsilon}} u_{\epsilon}\right| d x
$$

Remark that $\frac{\partial_{x} \mu\left(h_{\varepsilon}\right)}{\sqrt{h_{\varepsilon}}}=\partial_{x} \Psi\left(h_{\varepsilon}\right)$, and since $\partial_{x} \Psi\left(h_{\varepsilon}\right)$ is uniformly bounded in $L^{\infty}\left(0, T ; L^{2}(\Omega)\right)$, and $\sqrt{h_{\varepsilon}} u_{\epsilon}$ is uniformly bounded in $L^{2}\left(0, T ; L^{2}(\Omega)\right)$, then we get that $\mu\left(h_{\varepsilon}\right)$ is bounded in $L^{\infty}\left(0, T ; L^{1}(\Omega)\right)$. Concerning $\partial_{x} \mu\left(h_{\varepsilon}\right)$ we can write:

$$
\partial_{x} \mu\left(h_{\varepsilon}\right)=\frac{\partial_{x} \mu\left(h_{\varepsilon}\right)}{\sqrt{h_{\varepsilon}}} \sqrt{h_{\varepsilon}}=2 \sqrt{h_{\varepsilon}} \partial_{x} \Psi\left(h_{\varepsilon}\right)
$$

Using mass conservation, $\sqrt{h_{\varepsilon}}$ is in $L^{\infty}\left(0, T ; L^{2}(\Omega)\right)$, and so is $\partial_{x} \Psi\left(h_{\varepsilon}\right)$, which yields that $\partial_{x} \mu\left(h_{\varepsilon}\right)$ is in $L^{\infty}\left(0, T ; L^{1}(\Omega)\right)$. Therefore $\mu\left(h_{\varepsilon}\right)$ is in $L^{\infty}\left(0, T ; W^{1,1}(\Omega)\right)$, and eventually in $L^{\infty}(0, T ; \Omega)$, as $W^{1,1}(\Omega)$ is continuously injected in $L^{\infty}(\Omega)$. Lets get a further look on $\frac{d}{d t} \int_{\Omega} M\left(h_{\varepsilon}\right)$, in fact we can write

$$
\begin{aligned}
\int_{\Omega} \frac{d}{d t}=\int_{\Omega} M\left(h_{\varepsilon}\right) d x=\int_{\Omega} u_{\epsilon} \partial_{x} \mu\left(h_{\varepsilon}\right) d x & =-\int_{\Omega} \mu\left(h_{\varepsilon}\right) \partial_{x} u_{\epsilon} d x \\
& =-\int_{\Omega} \frac{\mu}{h_{\varepsilon}^{2}} h_{\varepsilon}^{2} \partial_{x} u_{\epsilon} \cdot d x \\
& =\int_{\Omega} h_{\varepsilon} \frac{\mu}{h_{\varepsilon}^{2}}\left(u_{\epsilon} \partial_{x} h_{\varepsilon}+\partial_{t} h_{\varepsilon}\right) d x \\
& =\int_{\Omega} h_{\varepsilon} u_{\epsilon} \partial_{x}\left(\int_{a}^{h_{\varepsilon}} \frac{\mu(s)}{s^{2}} d s\right)+h_{\varepsilon} \frac{\mu}{h_{\varepsilon}^{2}} \partial_{t} h_{\varepsilon} d x \\
& =\int_{\Omega}\left(\int_{a}^{h_{\varepsilon}} \frac{\mu(s)}{s^{2}} d s\right) \partial_{t} h_{\varepsilon}+h_{\varepsilon} \partial_{t}\left(\int_{0}^{h_{\varepsilon}} \frac{\mu(s)}{s^{2}} d s\right) d x \\
& =\frac{d}{d t} \int_{\Omega} h_{\varepsilon}\left(\int_{a}^{h_{\varepsilon}} \frac{\mu(s)}{s^{2}} d s\right) d x .
\end{aligned}
$$


And thus from 65], we get that $h_{\varepsilon}\left(\int_{a}^{h_{\varepsilon}} \frac{\mu(s)}{s^{2}} d s\right.$ is uniformly bounded in $L^{\infty}\left(0, T ; L^{1}(\Omega)\right)$. Again using assumptions on $\mu$ in 57)- 60 , we get that $h_{\varepsilon}\left(\int_{a}^{h_{\varepsilon}} \frac{\mu^{\prime}(s)}{s} d s\right.$ is bounded in $L^{\infty}\left(0, T ; L^{1}(\Omega)\right)$, and thus we get

$$
\|\mu\|_{L^{\infty}\left(0, T ; L^{1}(\Omega)\right)} \leq\left\|\int_{a}^{h_{\varepsilon}} \mu^{\prime}(s) d s\right\|_{L^{\infty}\left(0, T ; L^{1}(\Omega)\right)} \leq \| h_{\varepsilon}\left(\int_{a}^{h_{\varepsilon}} \frac{\mu^{\prime}(s)}{s} \|_{L^{\infty}\left(0, T ; L^{1}(\Omega)\right)}<\infty .\right.
$$

Remark 3.1. Using the assumptions (57)-(60), we can deduce that

$$
h_{\varepsilon}^{c_{1}} \leq \mu\left(h_{\varepsilon}\right) \leq h_{\varepsilon}^{c_{2}},
$$

and so we can deduce the uniform boundedness of $h_{\varepsilon}$ in $(0, T ; \Omega)$.

2-Information on $\partial_{t} \mu\left(h_{\varepsilon}\right)$. From above we have

$$
\partial_{t}\left(\mu\left(h_{\varepsilon}\right)\right)=-\partial_{x}\left(h_{\varepsilon} \mu^{\prime}\left(h_{\varepsilon}\right) u_{\epsilon}\right)+h_{\varepsilon} \mu^{\prime \prime}\left(h_{\varepsilon}\right) u_{\epsilon} \partial_{x} h_{\varepsilon}
$$

The second term is proven to be bounded in $L^{\infty}\left(0, T ; L^{1}(\Omega)\right)$. As for the first term, we have in fact $\left|h_{\varepsilon} \mu^{\prime}\left(h_{\varepsilon}\right) u_{\epsilon}\right| \leq$ $C\left|\mu\left(h_{\varepsilon}\right) u_{\epsilon}\right|$, hence lets check the boundedness of $\mu u_{\epsilon}$ :

$$
\mu\left(h_{\varepsilon}\right) u_{\epsilon}=\frac{\mu\left(h_{\varepsilon}\right)}{\sqrt{h_{\varepsilon}}} \sqrt{h_{\varepsilon}} u_{\epsilon} .
$$

Now for $h_{\varepsilon} \leq 1$, we have $h_{\varepsilon}^{a_{1}-\frac{1}{2}} \leq \frac{\mu\left(h_{\varepsilon}\right)}{\sqrt{h_{\varepsilon}}} \leq h_{\varepsilon}^{a_{2}-\frac{1}{2}}$, which is equivalent to have $h_{\varepsilon}^{\frac{1}{6}} \leq \frac{\mu\left(h_{\varepsilon}\right)}{\sqrt{h_{\varepsilon}}} \leq h_{\varepsilon}^{\frac{7}{2}} \leq 1$. And if $h_{\varepsilon} \geq 1$, then $\frac{\mu\left(h_{\varepsilon}\right)}{\sqrt{h e}} \leq \mu\left(h_{\varepsilon}\right)$. Hence, in all cases, $\frac{\mu}{\sqrt{h_{\varepsilon}}}$ is in $L^{\infty}\left(0, T ; L^{1}(\Omega)\right)$. Regard that

$$
\partial_{x}\left(\frac{\mu\left(h_{\varepsilon}\right)}{\sqrt{h_{\varepsilon}}}\right)=\frac{\partial_{x} \mu\left(h_{\varepsilon}\right)}{\sqrt{h_{\varepsilon}}}+\mu\left(h_{\varepsilon}\right) \partial_{x} \frac{1}{\sqrt{h_{\varepsilon}}}=2 \partial_{x} \Psi\left(h_{\varepsilon}\right)+\mu\left(h_{\varepsilon}\right) \partial_{x} \frac{1}{\sqrt{h_{\varepsilon}}}
$$

Hence

$$
\left|\partial_{x}\left(\frac{\mu\left(h_{\varepsilon}\right)}{\sqrt{h_{\varepsilon}}}\right)\right| \leq 2\left(\left|\partial_{x} \Psi\left(h_{\varepsilon}\right)\right|+\left|h_{\varepsilon} \mu^{\prime}\left(h_{\varepsilon}\right) \frac{\partial_{x} h_{\varepsilon}}{h_{\varepsilon}^{\frac{3}{2}}}\right| \leq C\left(\left|\partial_{x} \Psi\left(h_{\varepsilon}\right)\right|\right) .\right.
$$

Thus $\partial_{x}\left(\frac{\mu\left(h_{\varepsilon}\right)}{\sqrt{h_{\varepsilon}}}\right)$ is bounded in $L^{\infty}\left(0, T ; L^{2}(\Omega)\right) \subset L^{\infty}\left(0, T ; L^{1}(\Omega)\right)$, and therefore $\frac{\mu\left(h_{\varepsilon}\right)}{\sqrt{h_{\varepsilon}}}$ is bounded in $L^{\infty}\left(0, T ; W^{1,1}(\Omega)\right)$.

Now returning to $\mu\left(h_{\varepsilon}\right) u_{\epsilon}=\frac{\mu\left(h_{\varepsilon}\right)}{\sqrt{h_{\varepsilon}}} \sqrt{h_{\varepsilon}} u_{\epsilon}$, we deduce that $\mu\left(h_{\varepsilon}\right) u_{\epsilon}$ is bounded in $L^{2}\left(0, T ; L^{2}(\Omega)\right)$, and so is $h_{\varepsilon} \mu^{\prime}\left(h_{\varepsilon}\right) u_{\epsilon}$. Hence, $\partial_{x}\left(h_{\varepsilon} \mu^{\prime}\left(h_{\varepsilon}\right) u_{\epsilon}\right)$ belongs to $L^{2}\left(0, T ; W^{-1,1}(\Omega)\right)$. and so we can deduce the uniform boundedness of $\partial_{t} \mu\left(h_{\varepsilon}\right)$ in $L^{2}\left(0, T ; W^{-1,1}(\Omega)\right)$.

Convergence of $\mu$. As a result of the previous two parts, we got the following

$$
\mu \text { is uniformly bounded in } L^{\infty}\left(0, T ; W^{1,1}(\Omega)\right),
$$

$$
\begin{aligned}
& \partial_{t} \mu \text { is uniformly bounded in } L^{2}\left(0, T ; W^{-1,1}(\Omega)\right), \\
& \qquad W^{1,1}(\Omega) \subset \subset L^{2}(\Omega) \subset W^{-1,1}(\Omega) .
\end{aligned}
$$

Applying the compactness result of Aubin-Simon, we get the strong convergence of $\mu$ in $C\left(0, T, L^{2}(\Omega)\right)$.

Convergence of $h_{\varepsilon}$. Now we claim that $h_{\varepsilon}$ converges almost everywhere to some $\mathrm{h}$ such that the limit of $\mu\left(h_{\varepsilon}\right)$ is $\mu(h)$. This claim in fact is guaranteed using the fact that $\mu$ is continuous monotone function and thus invertible. On the other hand, we have from mass equation that $\partial_{t} h_{\varepsilon}=-\partial_{x}\left(\sqrt{h_{\varepsilon}} \sqrt{h_{\varepsilon}} u_{\epsilon}\right)$ is in $L^{2}\left(0, T ; L^{2}(\Omega)\right)$ 
since $\sqrt{h_{\varepsilon}}$ is bounded, and the bound on $\sqrt{h_{\varepsilon}} u_{\epsilon}$ follows from the estimations. Now regard that if $h_{\varepsilon} \leq 1$, then $h_{\varepsilon}$ is in $L^{\infty}\left(0, T ; L^{p}(\Omega)\right)$ for all $p>0$. If $h_{\varepsilon} \geq 1$, then from the assumptions on $\mu$ we have

$$
h_{\varepsilon}^{\frac{2}{3}} \leq \mu\left(h_{\varepsilon}\right) \leq h_{\varepsilon}^{4}, \quad \text { and } \quad c_{1} h_{\varepsilon} \mu^{\prime}\left(h_{\varepsilon}\right) \leq \mu\left(h_{\varepsilon}\right) \leq c_{2} h_{\varepsilon} \mu^{\prime}\left(h_{\varepsilon}\right) .
$$

Thus we get

which implies

$$
0 \leq \frac{1}{h_{\varepsilon}} \leq \frac{c \mu^{\prime}\left(h_{\varepsilon}\right) \sqrt{\mu\left(h_{\varepsilon}\right)}}{h_{\varepsilon}}=Z^{\prime}\left(h_{\varepsilon}\right)
$$

$$
\left|\partial_{x} \log h_{\varepsilon}\right|=\left|\frac{\partial_{x} h_{\varepsilon}}{h_{\varepsilon}}\right| \leq\left|\partial_{x} Z\left(h_{\varepsilon}\right)\right| \in L^{2}\left(0, T ; L^{2}(\Omega)\right) .
$$

But as $\partial_{x} h_{\varepsilon}=h_{\varepsilon} \partial_{x} \log h_{\varepsilon}$ and $h$ is bounded locally in time and space, then we deduce that $\partial_{x} h_{\varepsilon} \in L^{2}\left(0, T ; L^{2}(\Omega)\right)$. Hence, using Poincare Wirtinger inequality we deduce that $h_{\varepsilon}$ belongs to $L^{2}\left(0, T ; H^{1}(\Omega)\right)$. Finally applying compactness result, we get that $h_{\varepsilon}$ will converges strongly to some $h$ in $L^{2}\left(0, T ; L^{2}(\Omega)\right)$

Passage to the limit in the weak formulation. A weak formulation of the shallow water model (55) reads

$$
\int_{0}^{\infty} \int_{\Omega} h_{\varepsilon} \partial_{t} \psi d x d t+\int_{\Omega} h_{0}^{\varepsilon} \psi\left(h_{0}^{\varepsilon}\right) d x=-\int_{0}^{\infty} \int_{\Omega} h_{\varepsilon} u_{\epsilon} \partial_{x} \psi d x d t,
$$

and

$$
\begin{aligned}
& \varepsilon\left(\int_{0}^{\infty} \int_{\Omega} h_{\varepsilon} u_{\epsilon} \partial_{t} \phi d x d t\right.+\int_{\Omega} m_{0}^{\varepsilon} \phi(\cdot, 0) d x+\int_{0}^{\infty} \int_{\Omega} h_{\varepsilon} u_{\epsilon}^{2} \partial_{x} \phi d x d t \\
&\left.-\frac{8}{R_{e}} \int_{0}^{\infty} \int_{\Omega} h_{\varepsilon} \mu^{\prime}\left(h_{\varepsilon}\right) \partial_{x} u_{\epsilon} \partial_{x} \phi d x d t+\int_{0}^{\infty} \int_{\Omega} p\left(h_{\varepsilon}\right) \partial_{x} \phi d x d t\right) \\
&-\frac{1}{W_{e}} \int_{0}^{\infty} \int_{\Omega} h_{\varepsilon} \partial_{x}\left(\Psi^{\prime} \partial_{x}^{2} \Psi\right) \phi d x d t-\int_{0}^{\infty} \int_{\Omega} h_{\varepsilon} u_{\epsilon} \phi d x d t=0
\end{aligned}
$$

for all $\psi \in \mathcal{C}_{0}^{\infty}(\Omega \times[0, \infty))$ and $\phi \in \mathcal{C}_{0}^{\infty}(\Omega \times[0, \infty))$.

The obtained convergence results allow us to pass to the limit in the weak formulations. A dominated Lebesgue convergence theorem as well as the strong convergence of $h_{\varepsilon}$ and the weak convergence of the momentum in $L^{2}\left(0, T ; L^{2}(\Omega)\right)$ are used to pass to limit in all terms of the weak formulations except for the capillarity term. Concerning this latter, we will use the estimations on $Z(h)$ by showing the relation between $\Psi$ and $Z$ through the following identity:

$$
\left.h \partial_{x}\left(\Psi^{\prime} \partial_{x x} \Psi\right)=\partial_{x}\left(\frac{4 h_{\varepsilon} \mu^{\prime}}{\sqrt{\mu}} \partial_{x x} Z(h)\right)\right)-\partial_{x x}\left(\kappa(h) \partial_{x} Z(h)\right)+\partial_{x}\left(\kappa(h) \partial_{x x} Z(h)\right),
$$

where $\kappa(h)$ is a functional of $h$ given by

$$
\kappa(h)=\int_{0}^{h} \frac{2 s \mu^{\prime 2}}{\mu \sqrt{\mu}} d s .
$$

Now we can write the fifth term in the weak formulation as

$$
-\frac{1}{W_{e}} \int_{0}^{\infty} \int_{\Omega} h_{\varepsilon} \partial_{x}\left(\Psi^{\prime} \partial_{x}^{2} \Psi\right) \phi d x d t=\frac{1}{W_{e}} \int_{0}^{\infty} \int_{\Omega}\left(\frac{4 h_{\varepsilon} \mu^{\prime}}{\sqrt{\mu}}+\kappa\left(h_{\varepsilon}\right)\right) \partial_{x x} Z\left(h_{\varepsilon}\right) \partial_{x} \phi+\kappa\left(h_{\varepsilon}\right) \partial_{x} Z\left(h_{\varepsilon}\right) \partial_{x x} \phi d x d t .
$$

Lets have a look on the terms $\frac{4 h_{\varepsilon} \mu^{\prime}}{\sqrt{\mu}}$ and $\kappa\left(h_{\varepsilon}\right)$. In fact, both terms are positive quantities that can be treated in the same manner as $\sqrt{\mu}$. More precisely, using the assumptions (57)- 60) we've got

$$
\left|\frac{4 h_{\varepsilon} \mu^{\prime}\left(h_{\varepsilon}\right)}{\sqrt{\mu}}\right| \leq C|\sqrt{\mu}|
$$


But $\sqrt{\mu}$ belongs to $L^{\infty}\left(0, T ;(\Omega) \cap L^{\infty}\left(0, T ; L^{2}(\Omega)\right)\right.$. Thus, we infer the weak convergence of $\frac{4 h_{\varepsilon} \mu^{\prime}}{\sqrt{\mu}} \partial_{x x} Z\left(h_{\varepsilon}\right)$. The same applies to the remaining terms in $(3.1)$ since

$$
\kappa(h)=\int_{0}^{h} \frac{2 s \mu^{\prime}(s)^{2}}{\mu(s) \sqrt{\mu(s)}} d s \leq C \int_{0}^{h} \frac{\mu^{2}(s)}{\sqrt{\mu(s)}} d s=\sqrt{h_{\varepsilon}} .
$$

Thus we obtain the following weak formulation:

$$
\begin{gathered}
\int_{0}^{\infty} \int_{\Omega} h \partial_{t} \Psi(h) d x d t+\int_{\Omega} h_{0} \Psi\left(h_{0}\right) d x=-\int_{0}^{\infty} \int_{\Omega} h u \partial_{x} \Psi(h) d x d t \\
\frac{1}{W_{e}} \int_{0}^{\infty} \int_{\Omega}\left(\frac{4 h \mu^{\prime}(h)}{\sqrt{\mu(h)}}+\kappa(h)\right) \partial_{x x} Z(h) \partial_{x} \phi+\kappa(h) \partial_{x} Z(h) \partial_{x x} \phi d x d t-\int_{0}^{\infty} \int_{\Omega} h u \phi d x d t=0,
\end{gathered}
$$

which is the corresponding weak formulation of 63).

Remark. Note that the case $F(h)=h$ and $G(h)=\log h$ corresponds to the equation derived by Derrida, Lebowitz, Speer and Spohn describing several applications as in the fluctuation of a stationary non-equilibrium interface, and in zero-temperature zero-field approximation of quantum models for semi conductors. Solving such equation (in 1D and 2D) has been a great interest of mathematician and several results were obtained. As for the 1 dimensional case. a local in time classical solution was obtained in [4, and a nonnegative global weak solution was also proved to exist under several assumptions (Dirichlet-Neuman boundary condition, smallness of initial data,etc), see for instance [17]. Jungel and al. later in [13 proved existence of nonnegative global weak solution under a weaker condition on the initial data (measurability), and boundedness of the corresponding Lyapunov functional at time $t=0: \int u_{0}-\log u_{0}<\infty$. Their proof was based on regularizing the equation, using a semi-discrete system (in time), and compactness results to obtain convergence. Our method provides an other approach making the link between different models.

Acknowledgements. The authors acknowledge the ANR Project FRAISE managed by C. Ruyer-Quil.

\section{REFERENCES}

[1] F. Bernis, A. Friedman. Higher order nonlinear degenerate parabolic equations. J. Diff. Eqs, 83 (1), 179-206, (1990).

[2] A.L. Bertozzi, M. Pugh. The lubrication approximation for thin viscous films: the moving contact line with a porous media cut-off of van der Waals interactions. Nonlinearity, 7 (6), 1535, (1994).

[3] A.L. Bertozzi, M. Pugh. The lubrication approximation for thin viscous films: Regularity and long-time behavior of weak solutions. Com. Pure Appl. Math., 49(2), 85-123, (1996).

[4] P.M. Bleher, J.L Lebowitz, E.R Speer, H. Spohn. Existence and positivity of solutions of a fourth-order nonlinear PDE describing interface fluctuations. Com. Pure Appl. Math, 47(7), 923-942, (1994).

[5] D. Bresch, M. Colin, X. Lin, P. Noble. Lubrication Theory and Viscous Shallow-Water Equations, Recent Advances in PDEs: Analysis, Numerics and Control, 61-71. Springer (2018).

[6] D. Bresch, F. Couderc, P. Noble, J.-P. Vila. A generalization of the quantum Bohm identity: Hyperbolic CFL condition for Euler-Korteweg equations. C.R. Acad. Sciences Paris Volume 354, Issue 1, 39-43, (2016).

[7] D. Bresch, B. Desjardins. Existence of global weak solutions for 2D viscous shallow-water equations and convergence to the quasi-geostrophic model. Comm. Math. Phys. 238, 211-223 (2003).

[8] D. Bresch, B. Desjardins. Quelques modèles diffusifs capillaires de type Korteweg, C. R. Mécanique 332 (2004) $881-886$.

[9] D. Bresch, P.-E. Jabin. Viscous compressible flows under pressure. Fluid under pressure, 105-148. Eds T. Bodnar, G.P. Galdi, S. Necasova. Birkhauser (2019).

[10] D. Bresch, P. Noble. Mathematical derivation of viscous shallow-water equations with zero surface tension. Indiana Univ. Journal, Vol. 60, Number 4, 1137-1269 (2011).

[11] D. Bresch, A. Vasseur, C. Yu. Global existence of entropy-weak solutions to the compressible Navier-Stokes equations with non-linear density dependent viscosities. Submitted (2019).

[12] R. Dal Passo, L. Giacomelli, A. Shishkov. The thin film equation with nonlinear diffusion. Comm. Part. Different. Equat., 26, 1509-1557 (2001).

[13] J. Dolbeaut, I. Gentil, A. Jüngel. A logarithmic fourth-order parabolic equation and related logarithmic Sobolev inequalities. Communications in Mathematical Sciences, 4 (2), 275-290, (2006). 
[14] M.A. Fontelos, G. Kitavtsev, R.M. Taranets. Asymptotic decay and non-rupture of viscous sheets, Z. Angew. Math. Phys. 69-79, (2018).

[15] J.-F. Gerbeau, B. Perthame. Derivation of viscous Saint-Venant system for laminar shallow water: numerical validation. $D C D S-B$ volume 1, number 1, 89-102, (2001).

[16] G. Grün. On Free-Boundary problems arising in thin film flow. Habilitat. Thesis Univ. Bonn (2001).

[17] A. Jüngel, R. Pinneau, Global nonnegative solutions of a nonlinear fourth-order parabolic equation for quantum systems. SIAM Journal on Mathematical Analysis, 32 (3), 32 (4), 760-777, (2000).

[18] G. Kitavtsev, P. Laurençot, B. Niethammer, Weak solutions to lubrication equations in the presence of strong slippage, Methods Appl. Anal. 18 (2) (2011) 183-202.

[19] J. LI, Z.P. XIN. Global existence of weak solutions to the barotropic compressible Navier-Stokes flows with degenerate viscosities. arXiv:1504.06826 (2015)

[20] L.A Peletier, The porous media equation, Applications of nonlinear analysis in the physical sciences, 6, 229-241, (1981).

[21] I. Lacroix-Violet, A. Vasseur. Global weak solutions to the compressible quantum Navier-Stokes equation and its semiclassical limit. J. Math. Pures Appl. (9) 114, 191-210, (2018).

[22] H.-L. LI, J. LI, Z.P. XIN. Vanishing of vacuum states and blow-up phenomena of the compressible Navier-Stokes equations. Commun. Math. Phys. 281 (2008) 401-444.

[23] A. Münch, B.A. Wagner, T.P. Witelski. Lubrication models with small to large slip lengths. J. Eng. Math. 53 (3-4) (2005) 359-383.

[24] A. VAsseur, C. Yu. Existence of global weak solutions for 3D degenerate compressible Navier-Stokes equations. Invent. Math. 206, no. 3, 935-974, (2016). 